\title{
Dynamic crossflow filtration with a rotating tubular membrane: Using centripetal force to decrease fouling by buoyant particles
}

Submitted for consideration as a publication in the Chemical Engineering Research and Design journal

Pengyu Ji ${ }^{1}$, Abdul Motin ${ }^{2}$, Wenqian Shan ${ }^{3}$, André Bénard ${ }^{2}$, Merlin L. Bruening ${ }^{1}$, Volodymyr V. Tarabara ${ }^{3 *}$

June 8, 2015

1 Department of Chemistry, Michigan State University, East Lansing, MI 48824, USA

2 Department of Mechanical Engineering, Michigan State University, East Lansing, MI 48824, USA

3 Department of Civil and Environmental Engineering, Michigan State University, East Lansing, MI 48824, USA

"Corresponding author: Phone: +1 (517) 432-1755; Fax: +1 (517) 432-1827; Email: tarabara@msu.edu 


\section{Abstract}

This paper describes a dynamic membrane filtration system that combines crossflow filtration and centrifugal separation in a rotating tubular membrane. Because of the noslip boundary condition, membrane rotation leads to higher centripetal force near the lumen wall than introduction of a rotating flow. In this fundamental exploratory study, hollow glass microspheres ( 5 to $35 \mu \mathrm{m}$ diameters) serve as model low-density, separate-phase foulants during filtration of aqueous suspensions through a tubular ceramic membrane (nominal $0.14 \mu \mathrm{m}$ pore size). At low crossflow rates, membrane rotation at $1725 \mathrm{rpm}$ decreases fouling and shifts the microsphere size distribution in the membrane cake towards smaller diameters. Force balance calculations suggest that centripetal force should move particles with diameters $>\sim 17 \mu \mathrm{m}$ away from the lumen surface. Moreover, azimuthal and longitudinal shear stresses will also selectively remove larger particles from the membrane cake. Computational fluid dynamics (CFD) simulations show that the rotational flow does not fully develop in the membrane lumen and the fluid radial velocity peaks before the membrane wall. Both of these factors will decrease movement of low-density particles away from the lumen wall. Nevertheless, consistent with experimental data, CFD simulations show greatly decreasing encounters of particles with the membrane wall as particle size increases.

Keywords: dynamic crossflow filtration; ceramic membrane; computational fluid dynamics; fouling; rotating membrane 


\section{Introduction}

Crossflow filtration is a membrane-based separation technique with many applications including desalination (Lee et al., 2011), wastewater treatment (Carstensen et al., 2012; Le-Clech et al., 2006), beverage production (El Rayess et al., 2011; van der Sman et al., 2012), and hemodialysis (Nie et al., 2015). In contrast to conventional crossflow filtration where liquid flows along stationary membrane surfaces to reduce concentration polarization or cake formation, dynamic crossflow filtration (DCF, also called dynamic filtration or shear-enhanced filtration) involves movement of solid boundaries such as the membrane itself or other surfaces positioned in the membrane's proximity (Jaffrin, 2008, 2012). Importantly, dynamic shear-enhanced filtration can decouple crossflow velocity and shear to control them independently (Jaffrin, 2008).

Since its conceptual introduction in the late 1960s, DCF has been proposed or applied to separate a broad range of feeds including oil-in-water emulsions (Li et al., 2009; Reed et al., 1997; Viadero Jr et al., 2000) (milk, in particular (Ding et al., 2003; Frappart et al., 2006)); suspensions of latex particles (Mikulášek and Doleček, 1994), glass microspheres (Engler and Wiesner, 2000), yeast (Brou et al., 2002; Lee et al., 1995; Liu et al., 2012), bacterial cells (Frenander and Jönsson, 1996; Kroner and Nissinen, 1988), algae (Ochirkhuyag et al., 2008), and clay (Liu et al., 2012); black liquor (Bhattacharjee and Bhattacharya, 2006); aqueous slurries of $\mathrm{SiO}_{2}$ (Choi et al., 1999; Engler and Wiesner, 2000) and $\mathrm{CaCO}_{3}$ (He et al., 2007; Liu et al., 2012; Tu and Ding, 2010); protein solutions (Holeschovsky and Cooney, 1991); pulp (Ochirkhuyag et al., 2008); and navy ship wastewater (Bendick et al., 2014). Although desalination by reverse and forward osmosis (Sherwood et al., 1967) was the initial DCF target and some studies utilized nanofiltration membranes (e.g. (Frappart et al., 2006)), most DCF developments relate to porous membranes for ultrafiltration (Bhattacharjee and Bhattacharya, 2006; Ding et al., 2003; Hallstrom and Lopez-Leiva, 1978; Holeschovsky and Cooney, 1991; Li et al., 2009; Reed et al., 1997; Viadero Jr et al., 2000) and 
microfiltration (Aubert et al., 1993; Brou et al., 2002; Engler and Wiesner, 2000; Espina et al., 2008; He et al., 2007; Lee et al., 1995; Li et al., 2009; Mikulášek and Doleček, 1994; Tu and Ding, 2010).

Typical DCF processes employ vibration (Beier et al., 2006; Genkin et al., 2006) or rotation of disks (Bouzerar et al., 2000; Brou et al., 2002; Ding et al., 2003; Ding et al., 2006; Espina et al., 2008; Frappart et al., 2006; Frenander and Jönsson, 1996; He et al., 2007; Lee et al., 1995; Li et al., 2009; Mänttäri et al., 2006; Sen et al., 2010; Tu and Ding, 2010) or membranes (Aubert et al., 1993; Beaudoin and Jaffrin, 1989; Belfort et al., 1993a; Belfort et al., 1993b; Bendick et al., 2014; Bhattacharjee and Bhattacharya, 2006; Choi et al., 1999; Dolecek et al., 1995 ; Engler and Wiesner, 2000; Hallstrom and Lopez-Leiva, 1978; Holeschovsky and Cooney, 1991; Kaplan and Halley, 1990; Kroner and Nissinen, 1988; Kroner et al., 1987; Liu et al., 2012; Mikulášek and Doleček, 1994; Murase et al., 1991; Ochirkhuyag et al., 2008; Park et al., 1994; Reed et al., 1997; Rock et al., 1986; Sarkar et al., 2011; Sen et al., 2010; Serra and Wiesner, 2000 ; Sherwood et al., 1967; Viadero Jr et al., 2000; Vigo et al., 1985). The disks rotate in close proximity to a stationary membrane and can include multiple shafts (Ding et al., 2006; Espina et al., 2008; He et al., 2007; Tu and Ding, 2010) and vanes (Ding et al., 2003; Li et al., 2009; Sen et al., 2010). The family of rotating membrane systems includes an important subgroup of rotating annular filters (Belfort et al., 1993a; Belfort et al., 1993b; Choi et al., 1999; Dolecek et al., 1995; Hallstrom and Lopez-Leiva, 1978; Holeschovsky and Cooney, 1991; Kroner and Nissinen, 1988; Kroner et al., 1987; Mikulášek and Doleček, 1994; Murase et al., 1991; Park et al., 1994; Sherwood et al., 1967; Vigo et al., 1985). In these filters, the feed flows through the annular gap between two concentric cylinders, where the porous membrane is the inner rotating cylinder and an impermeable cylinder is the outer and stationary wall; Taylor vortices that form in such flow channels can greatly enhance membrane performance as demonstrated in applications ranging from blood plasma filtration (Rock et al., 1986) to ultrafiltration of cutting oil emulsions (Vigo et al., 1985). Several studies also employed flat membranes in DCF (Aubert et al., 1993; Bendick et al., 2014; Bhattacharjee and Bhattacharya, 2006; 
Engler and Wiesner, 2000; Ochirkhuyag et al., 2008; Reed et al., 1997; Sarkar et al., 2011; Sen et al., 2010; Serra and Wiesner, 2000 ; Viadero Jr et al., 2000); in such systems, a membrane mounted on a porous support rotates next to a wall that is stationary or rotating in the opposite direction to enhance shear. Other configurations such as a rotating helical membrane (Liu et al., 2012) and a rotating impermeable tube just upstream of a stationary tubular membrane (Shan, 2010; Shan et al., 2010) have been explored as well.

DCF studies to date focused on increasing wall shear stress and turbulence, or on employing local hydrodynamics (e. g. Taylor vortices in rotating annual filters) to minimize concentrate polarization and cake formation. Although rotating disks can enhance separation of oil-water mixtures, the centripetal forces in such systems are directed along and not normal to, the membrane surface. In rotating annular filters centripetal forces should help move the dispersed lighter phase away from the inner cylindrical membrane wall, but to our knowledge the importance of the buoyant force for separation has not been studied. This paper builds on our earlier experimental (Benard et al., 2014; Shan et al., 2010; Tarabara et al., 2013) and computational modeling work (Motin et al., 2015) and proposes a DCF system that combines crossflow filtration and centrifugal separation (Figure 1) where the density difference between the continuous and dispersed phases leads to particle transport away from the membrane. A rotating cylindrical membrane operates in an inside-out filtration to minimize deposition of the buoyant particles of the dispersed phase on the lumen surface. This study examines how deposition on the membrane varies with particle size to investigate the limits of membrane rotation for minimizing fouling through buoyancy forces. These limits will suggest whether such a strategy might apply to separation of oil-water emulsions. 


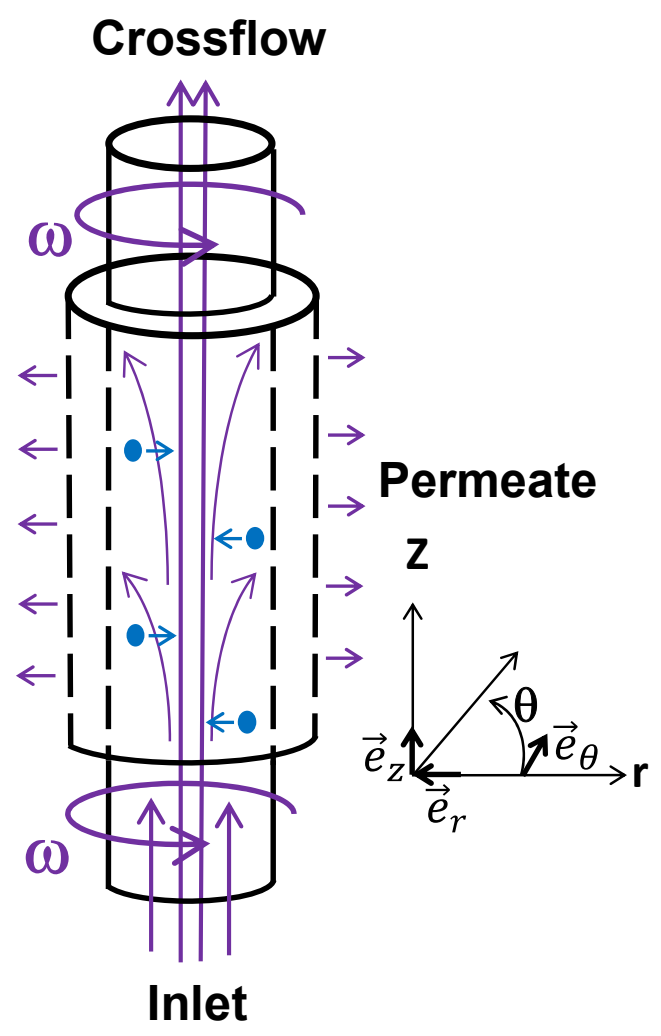

Figure 1: Diagram of crossflow filtration of a particle suspension through a rotating, solvent-permeable membrane.

\section{Approach}

\subsection{Crossflow filtration with a rotating membrane: Separation principle}

In a conventional centrifugation, the solution or suspension spins with an angular velocity $\omega=\frac{d \theta}{d t}=\frac{v_{l, \theta}}{r}$, where $v_{l, \theta} \vec{e}_{\theta}$ is the azimuthal velocity $\left(\vec{e}_{\theta}\right.$ is the azimuthal unit vector) of the liquid. In a rotating flow field, particles experience a net force, $\vec{F}$, which is the sum of the centripetal force, $\vec{F}_{c}$, and the buoyancy force, $\vec{F}_{b}$ (Figure 2A). Eq. (1) gives the sum of these opposing forces that act along $\vec{e}_{r}$, the unit radial vector pointing toward the axis of rotation, on a spherical particle of diameter $d_{p}$ in a fluid of density $\rho_{l}$. 


$$
\vec{F}=\vec{F}_{b}+\vec{F}_{c}=\frac{\pi d_{p}^{3}}{6}\left(\rho_{l}-\rho_{p}\right) \frac{v_{l, \theta}^{2}}{r} \vec{e}_{r}
$$

In this equation, $\rho_{p}$ is particle density and $r$ is the distance between the particle and the axis of rotation. For a negatively buoyant particle, this force is positive and acts toward the axis of rotation. Particles moving toward the center with a velocity $v_{p, r} \vec{e}_{r}$ also experience a drag force, $\vec{F}_{D}$, which for a spherical particle in the creeping flow regime (that is at a low particle Reynolds number $R e_{p}=\frac{v_{p} d_{p} \rho_{l}}{\mu_{l}} \ll 1$ ) is given by Stokes law:

$F_{D}=-3 \mu_{l} \pi d_{p} v_{p, r} \vec{e}_{r}$

where $\mu_{l}$ is the solution viscosity. Setting the sum of $\vec{F}$ and $\vec{F}_{D}$ equal to zero and solving for $v_{p, r}$ gives Stokes law:

$v_{p, r}=\frac{d_{p}^{2}}{18 \mu}\left(\rho_{l}-\rho_{p}\right) \frac{v_{l, \theta}^{2}}{r}=\frac{d_{p}^{2}}{18 \mu} \Delta \rho \omega^{2} r$

Larger density differences $(\Delta \rho)$ and rotational velocities $\left(v_{l, \theta}\right.$ or $\left.\omega\right)$ translate into larger particle velocities $\left(v_{p, r}\right)$ and better separations. If the rotating wall is porous (Figures $2 \mathrm{~B}$ and $2 \mathrm{C}$ ), water flux through the wall will change the radial velocity of the particles with respect to the solvent. For a particle this will alter the drag force by $\vec{F}_{j}$ :

$\vec{F}_{j}=-3 \mu \pi d_{p} v_{l, r} \vec{e}_{r}$

where $v_{l, r} \vec{e}_{r}$ is the velocity of the continuous phase in the radial direction. At the lumen surface (i. e. for $r=R$ ), $v_{l, r}=-j$, where $j$ is the permeate flux defined as permeate flow rate $\left(\mathrm{m}^{3} / \mathrm{s}\right)$ divided by the surface area of the lumen wall.

Summing the forces in eqs. (1), (2) and (4) and setting them equal to zero leads to eq. (5) for the radial velocity, $v_{p, r} \vec{e}_{r}$, of a particle suspended inside the channel of a rotating tube with porous walls (e. g. a membrane) in the presence of flow through the wall. 
A

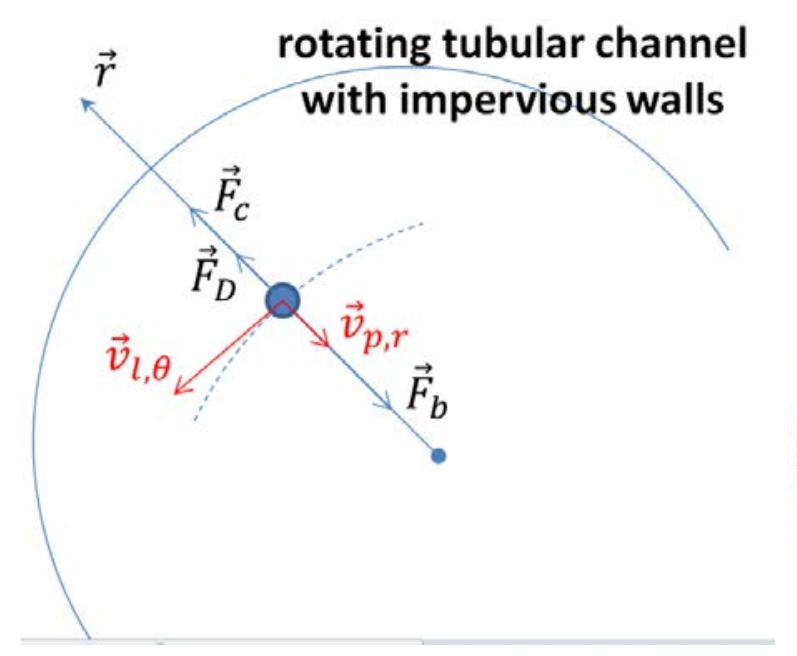

B

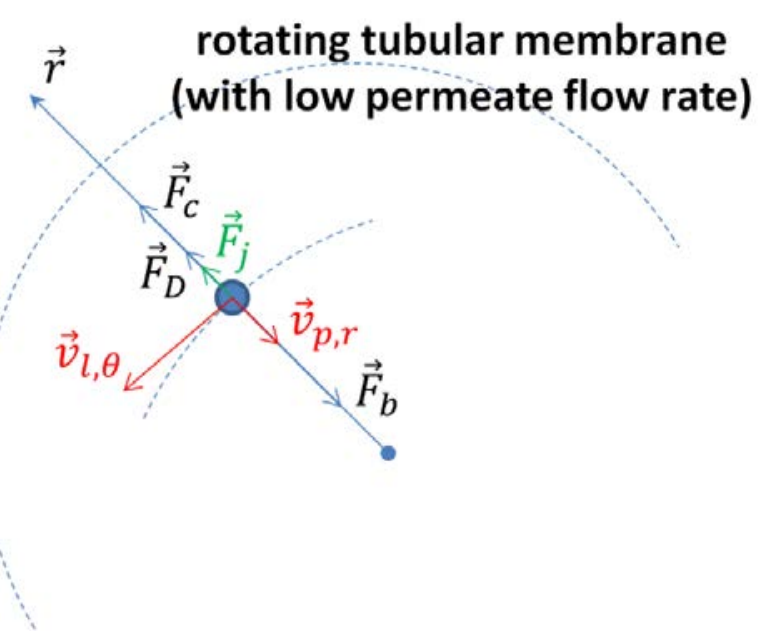

C

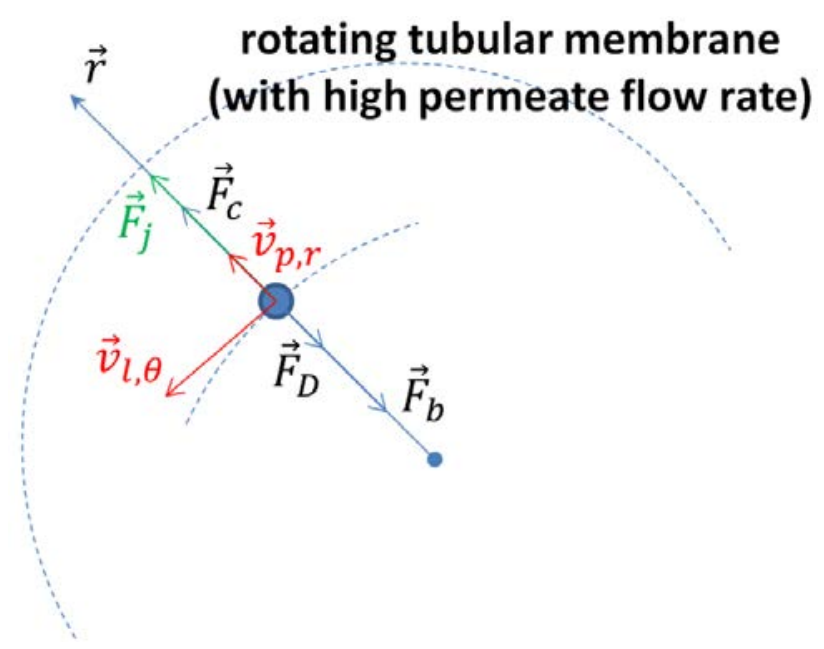

Figure 2: Force balance in a radial direction on a suspended negatively buoyant particle in a tubular channel with impervious walls $(A)$ and in tubular membranes in the presence of permeate flow (B, C). As the rate of permeation through the membrane increases beyond a critical value, the direction of particle movement reverses from centripetal (B) to centrifugal (C). 
The sign and magnitude of $v_{p, r}$ depend on $j, d_{p}, \Delta \rho$ and the azimuthal velocity $v_{l, \theta}$ :

$v_{p, r} \vec{e}_{r}=\left(\frac{d_{p}^{2}}{18 \mu} \Delta \rho \frac{v_{l, \theta}^{2}}{r}-j\right) \vec{e}_{r}$

If the product of $d_{p}^{2}, \Delta \rho$, and $v_{l, \theta}^{2}$ is sufficiently large and $j$ is sufficiently small, $v_{p, r}$ will be positive and the particles will move towards the axis of rotation (Figure 2B, we assume the particles are less dense than the liquid.) If $j$ is sufficiently large, particles will move toward the porous wall. Thus, in crossflow filtration with a rotating tubular membrane, large particles will move towards the center of the membrane channel, but small particles may move to the membrane surface with the permeate flow. For large particles, their movement toward the center of the membrane should decrease or prevent fouling of the membrane surface with these particles.

\subsection{Flow profiles in rotating membranes with crossflow}

In traditional rotating flow obtained with a mixer or baffle and a stationary cylinder wall, the azimuthal velocity of the outmost layer of liquid is zero due to the no-slip boundary condition (Figure $3 \mathrm{~A}$ ). Thus, the fluid azimuthal velocity initially increases with radial distance from the center of the cross section, reaches a maximum value, and drops to zero at the wall. In contrast, our filtration system uses a rotating membrane to induce the swirling motion of the continuous phase. If the fluid mixture rotates as a block inside the membrane, the angular velocity $(\omega)$ is the same for all objects regardless of their position, but the azimuthal velocity $\left(v_{l, \theta}=\omega r\right)$ of the fluid varies with the distance from the center of rotation. Importantly, the outermost layer of liquid near the membrane wall should have the largest azimuthal velocity, which is equal to $\omega R$, where $R$ is the radius of the tubular membrane (Figure 3B).

This high value of $v_{l, \theta}$ at the wall should help minimize fouling by moving low density particles away from the wall. Based on the flow profile within the rotating membrane, 
we expect that larger particles will concentrate near the center of the membrane under the action of centripetal force and pass through the system with the crossflow. Permeate flow may drag small particles to the membrane surface, but the membrane will reject such particles when they are larger than the membrane pore size. In principle, the rotating membrane should foul more slowly than a stationary one.
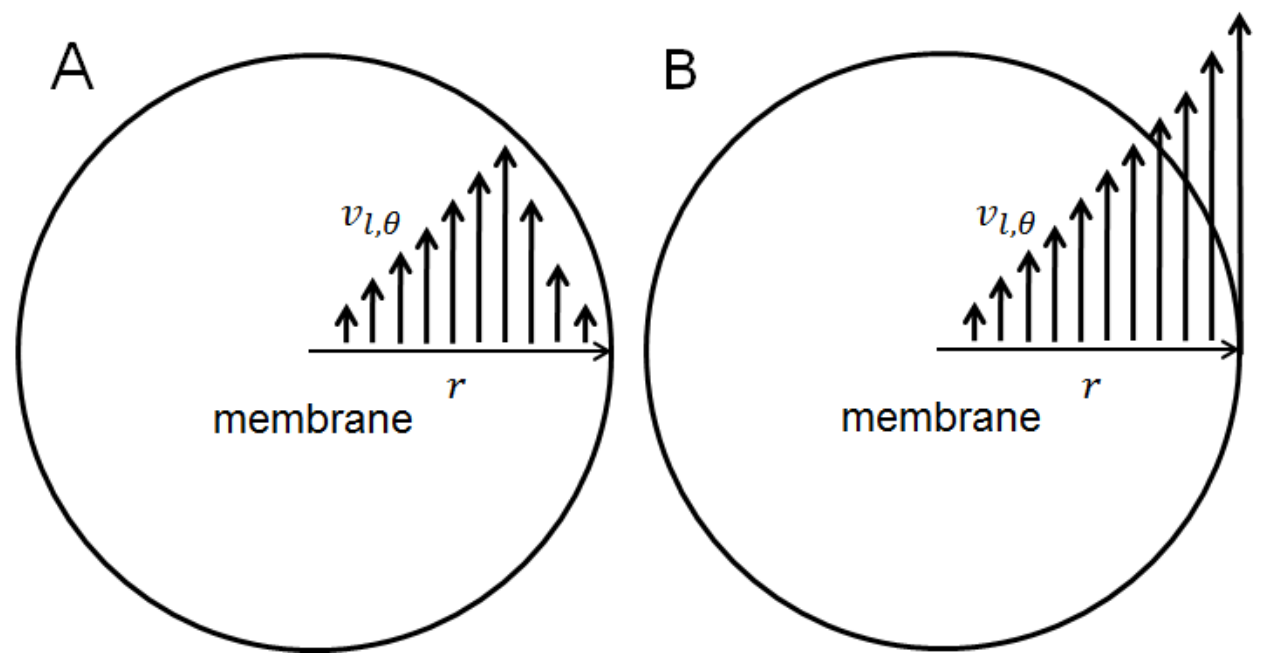

Figure 3: Conceptual radial distribution of azimuthal velocities, $v_{l, \theta}(r)$, for rotating flow inside (A) a stationary membrane and $(B)$ a rotating membrane. In the former case, flow rotation stems from introducing the feed into the membrane channel under an angle. In the latter case, the flow enters the membrane channel co-axially with the channel, and momentum transfer from the rotating wall rotates the fluid. The depicted velocity profiles are idealizations; Figure $8 \mathrm{c}$ shows more realistic velocity profiles for the rotating membrane.

\subsection{Erosion of the membrane cake}

In a net depositional environment, erosion due to flow tangential to the deposit's surface can have a significant impact on the rate of deposit accumulation. Lu and Ju (Lu and Ju, 1989) considered the moment of hydrodynamic forces acting on a particle about its point of contact with a cake surface as a criterion of whether the particle remains pinned on the surface or is swept off. Figure 4 illustrates this criterion for a rotating membrane. The moment due to the sum of forces acting tangentially to the membrane surface, $\vec{F}_{t o t}^{\tau}$, 
around the point $\mathrm{A}$ is $\left\|\vec{F}_{\text {tot }}^{\tau}\right\| \frac{d_{p}}{2} \cos \theta$ (presuming the net force points upward), where $\theta$ is the angle of repose (defined as shown in Figure 4). Similarly, the moment due to the sum of forces acting normal to the membrane surface, $\vec{F}_{\text {tot }}^{n}$, around point $A$ is $-\left\|\vec{F}_{t o t}^{\tau n}\right\| \frac{d_{p}}{2} \sin \theta$. Because $\vec{F}_{\text {tot }}^{n}$ points toward the lumen surface for any particles that remain there, the moment due to the normal forces is negative, and eq. (6) gives the sum of moments:

$M=\frac{d_{p}}{2}\left(\left\|\vec{F}_{\text {tot }}^{\tau}\right\| \cos \theta-\left\|\vec{F}_{\text {tot }}^{n}\right\| \sin \theta\right)$

$\vec{F}_{\text {tot }}^{\tau}$ is the sum of the drag force, $\vec{F}_{D}^{\tau}$, the gravitational buoyancy force, $\vec{F}_{b}^{\tau}$, and the gravity force, $\vec{F}_{g}$, which points downward:

$\vec{F}_{t o t}^{\tau}=\vec{F}_{D}^{\tau}+\vec{F}_{b}^{\tau}+\vec{F}_{g}$

The drag force can be approximated using a modified Stokes equation:

$\vec{F}_{D}^{\tau}=C_{1} 3 \pi \mu d_{p}\left[v_{l, z} \vec{e}_{z}\right]_{r=R-\frac{d_{p}}{2}}$

where the axial liquid velocity, $v_{l, z} \vec{e}_{z}$, is evaluated as the Hagen-Poiseuille velocity at the center of the particle (i.e. at a distance $\frac{d_{p}}{2}$ away from the membrane wall) and $C_{1}=1.0009$ is a coefficient that accounts for the presence of the membrane (O'Neill, 1968).

The gravity-based buoyancy force, $\vec{F}_{b}^{\tau}=\frac{\pi d_{p}^{3}}{6} \rho_{l} g \vec{e}_{z}$, and the gravity force, $\vec{F}_{g}=-\frac{\pi d_{p}^{3}}{6} \rho_{p} g \vec{e}_{z}$, are directed tangentially to the membrane surface because the membrane in our experiments is positioned vertically. The force $\vec{F}_{\text {tot }}^{n}$ is the sum of all hydrodynamic forces that act on the particle in the direction normal to the membrane surface: 


$$
\vec{F}_{t o t}^{n}=\vec{F}_{c}+\vec{F}_{b}^{n}+\vec{F}_{j}
$$

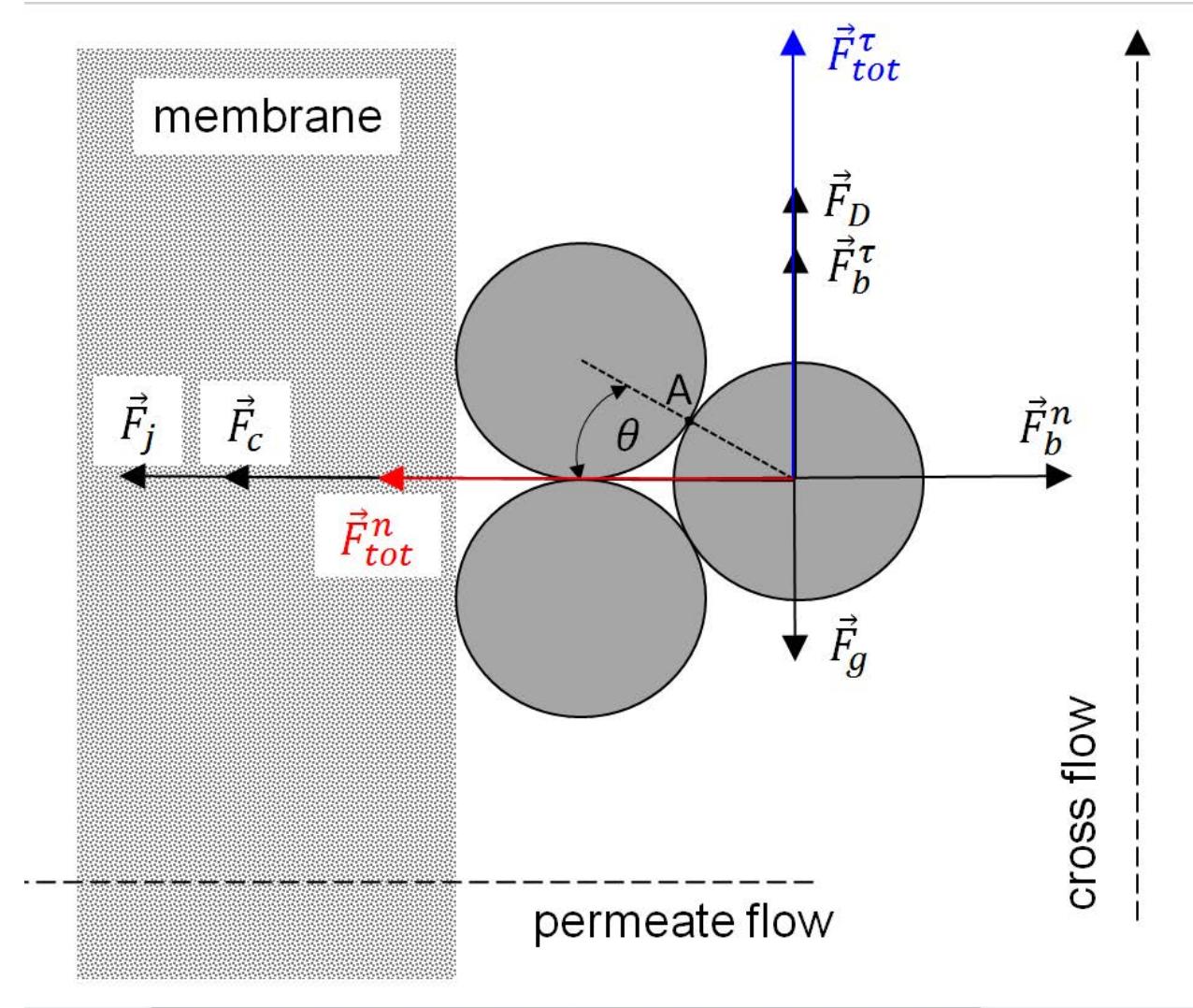

Figure 4: Hydrodynamic forces acting on a buoyant microsphere positioned on a deposit of similar particles at the surface of a rotating crossflow filtration membrane. See the text for the definitions of forces.

The gravity-based buoyancy force, $\vec{F}_{b}^{\tau}=\frac{\pi d_{p}^{3}}{6} \rho_{l} g \vec{e}_{z}$, and the gravity force, $\vec{F}_{g}=-\frac{\pi d_{p}^{3}}{6} \rho_{p} g \vec{e}_{z}$, are directed tangentially to the membrane surface because the membrane in our experiments is positioned vertically.

Eq. (9) does not include XDLVO forces; for the very large particles employed in our study van-der-Waals forces are negligible and the effect of electrostatic repulsion does 
not extend beyond a distance that is $\sim 10^{3}$ times smaller than particle radius. Eq. (1) gives and expression for $\vec{F}_{b}^{n}+\vec{F}_{c}$, and $\vec{F}_{j}$ is the drag force exerted on the particle touching the membrane surface by the flow permeating the membrane. $\vec{F}_{j}$ is given by:

$\vec{F}_{j}=\phi 3 \mu_{l} \pi d_{p}\left(j-v_{p, l i f t}\right) \vec{e}_{r}$

which is Stokes law modified to account for the inertial lift force and to include the wall correction factor, $\phi$, derived by Sherwood (Sherwood, 1988):

$\phi=0.36\left(\frac{\delta_{m}}{R_{m}} \frac{4}{d_{p}^{2}}\right)^{-\frac{2}{5}}$

In eq. (11), $\delta_{m}$ and $R_{m}$ are the total thickness and the total hydraulic resistance, respectively, of the membrane. In our case, the resistance of the cake is negligible compared to the resistance of the membrane. The lift velocity, $v_{p, l i f t}$, is given by (Saffman, 1956; Vasseur and Cox, 1976)

$\vec{v}_{p, l i f t}=-\frac{61 \rho_{l}}{567 \mu_{l}} \dot{\gamma}_{w}^{2}\left(\frac{d_{p}}{2}\right)^{3} \vec{e}_{r}=\frac{61 \rho_{l}}{567 \mu_{l}}\left(4 \frac{\bar{v}_{l, z}}{R}\right)^{2}\left(\frac{d_{p}}{2}\right)^{3} \vec{e}_{r}$

where $\dot{\gamma}_{w}$ is the shear rate at the wall. Approximating flow in the membrane channel using the Hagen-Poiseuille equation gives the following expression for the wall shear rate:

$\dot{\gamma}_{w}=\left[\frac{d v_{z}}{d r}\right]_{r=R}=4 \frac{\bar{v}_{l, z}}{R}$

where $\bar{v}_{l, z}$ is the average crossflow velocity in the membrane channel $\left(\bar{v}_{l, z}=0.132 \mathrm{~m} / \mathrm{s}\right)$. A negative value of the moment given by eq. (6) indicates that the particle will remain on the surface, and eq. (14) corresponds to the zero value of the net moment.

$\left\|\vec{F}_{\text {tot }}^{\tau}\right\|=\left\|\vec{F}_{\text {tot }}^{n}\right\| \tan \theta$

Solving eq. (14) for the particle diameter will yield the cut-diameter, which is the diameter of the largest particle that remains pinned on the surface. 


\subsection{Mass balance on particles in crossflow filtration with erosion}

In its most general form the mass balance on particles in the crossflow membrane channel with complete particle rejection is:

$Q_{f} C_{f}=Q_{r} C_{r}+\dot{m}_{\text {cake }}^{\text {net }}$

where $\dot{m}_{\text {cake }}^{\text {net }}$ is the net particle deposition rate. Not all deposited particles will remain in the cake. Some of the deposited material will exit with the retentate stream due to reentrainment by the crossflow and or as part of the flowing sub-layer of the cake (Davis and Birdsell, 1987; Leonard and Vassiliefl, 1984). To describe this process we introduce the erosion parameter, $\kappa(0<\kappa<1)$ and rewrite the mass balance as:

$Q_{f} C_{f}=Q_{r} C_{r}+(1-\kappa) \dot{m}_{\text {cake }}$

In this equation, $\dot{m}_{\text {cake }}$ represents deposition only, and the factor of $(1-\kappa)$ takes erosion into account. When $\kappa=0$ no erosion occurs whereas $\kappa=1$ describes full erosion when cake does not accumulate and all particles that enter the membrane channel exit it with the retentate flow. In the creeping flow regime $\left(R e_{p}<1\right)$, the drag force on a spherical particle is proportional to the particle's size, $d_{p}$. Thus crossflow should preferentially remove larger particles from the cake. The previous section and prior studies (e. g. (Lu and Ju, 1989; Ould-Dris et al., 2000)) suggest a critical particle size for erosion, so we propose the following empirical expression for the erosion parameter:

$\kappa=1-\exp \left(-\frac{d_{p}-\delta_{e}}{\delta_{e}}\right)$, for $d_{p} \geq \delta_{e}$

$\kappa=0$, for $d_{p}<\delta_{e}$

where $\delta_{e}$ is a constant with the unit of length, which approximately corresponds to the cut-diameter, i. e. when $d_{p}=\delta_{e}$ there is no erosion. The functional form of eq. (17a) ensures that $\kappa$ is bound within the $[0 ; 1]$ interval. 


\subsection{Separation efficiency. Total and reduced grade efficiencies}

Figures of merit for standard particle separation equipment, such as hydrocyclones, include the total grade efficiency, $G_{T}$, and the reduced grade efficiency, $G_{R}$ (Svarovsky and Thew, 1992). In the present case, $G_{T}$ is simply the ratio of the mass flow rate of particles exiting the membrane channel with the retentate to the total mass flow rate of particles entering the membrane channel at the inlet. Grade efficiencies approaching unity indicate a low rate of particles sticking to the membrane wall. However, this measure does not take into account the relative volumes of permeate and retentate flows. In contrast, $G_{R}$ includes the ratio, $R_{f}$, of the retentate flow rate, $Q_{r}$, to the feed flow rate, $Q_{F}$ :

$G_{R}=\frac{G_{T}-R_{f}}{1-R_{f}}$

$R_{f}=\frac{Q_{r}}{Q_{F}}$

Eq. (19) gives an equivalent definition of $G_{R}$ in terms of the concentration of particles in the feed, $C_{f}$, and the concentration of accumulating particles, $C_{w}$, in the solution flowing through the membrane wall. (See Supplementary Material (SM) for the derivation of eq. (19)).

$G_{R}=\frac{C_{f}-C_{w}}{C_{f}}$

Note that $C_{w}$ is not the concentration of particles in the permeate, but the concentration of particles that would exist in the permeate if the particles accumulating on the membrane wall passed through it. The actual concentration of particles in the permeate is zero because of the small membrane pore size.

Without taking into account erosion, the definition of $G_{T}$ (eq. 18a) and the mass balance (eq. (16) with $\kappa=0$ ) lead to 
$G_{T}^{\text {no erosion }}=\frac{\dot{m}_{r}}{\dot{m}_{f}}=\frac{Q_{f} C_{f}-\dot{m}_{\text {cake }}}{Q_{f} C_{f}}$

Including erosion gives

$G_{T}^{\text {erosion }}=\frac{\dot{m}_{r}}{\dot{m}_{f}}=\frac{Q_{f} C_{f}-(1-\kappa) \dot{m}_{\text {cake }}}{Q_{f} C_{f}}$

It can be easily shown that

$G_{T}^{\text {erosion }}=1-(1-\kappa)\left(1-G_{T}^{\text {no erosion }}\right)$

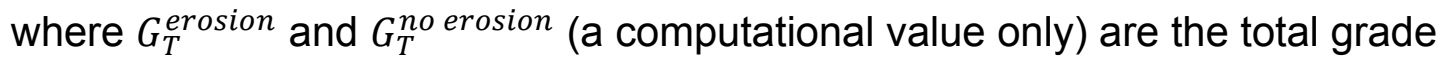
efficiencies with and without taking into account erosion, respectively. By definition, eq. (22) gives the reduced grade efficiency of a crossflow membrane system in the presence of erosion

$$
G_{R}^{\text {erosion }}=\frac{G_{T}^{\text {erosion }}-R_{f}}{1-R_{f}}
$$

\subsection{Numerical simulation approach}

The numerical simulation was performed for a feed Reynolds number of 1270 (feed inlet velocity is $0.21 \mathrm{~m} / \mathrm{s}$ ), an angular membrane velocity of $1725 \mathrm{rpm}$, a permeate water flux of $3200 \mathrm{~L} /\left(\mathrm{m}^{2} \cdot \mathrm{h}\right)$, and a transmembrane pressure of $0.1 \mathrm{MPa}$. The pressure and velocity of the continuous (liquid) phase were predicted by solving the Navier-Stokes equations (eq. (23)) using the Eulerian approach. The Navier-Stokes equations for steady and incompressible flow relative to the frame of reference rotating with an angular velocity $\omega$, are given by

$\nabla \cdot \vec{v}_{l}=0$

and

$\vec{v}_{l} \cdot \nabla \vec{v}_{l}=-\frac{1}{\rho} \nabla P-\omega^{2} \vec{r}+\nu \nabla^{2} \vec{v}_{l}+\vec{S}$

where $\vec{v}_{l}$ denotes the velocity vector of liquid relative to the rotating frame of reference. The term $\omega^{2} \vec{r}$ accounts for the rotation of the membrane and is often called the "centrifugal force"; it is effective in the filter domain and equals zero in the bulk flow 
domain. The position vector $\vec{r}$ locates arbitrary points in the computational domain relative to the origin of the rotating frame of reference. In eq. (23), $\vec{S}$ is the additional source term in the filter domain; this source term is modeled based on Darcy's law (see (Motin et al., 2013; Motin et al., 2015) for details).

The motion of the dispersed phase is estimated by tracking the trajectories of particles through the calculated flow field using a Lagrangian approach. A group of $N$ monodisperse particles of density $0.46 \mathrm{~g} / \mathrm{mL}$ (mimicking hollow glass microspheres; see section 3.1) are introduced in the inlet plane. Particles are considered as not interacting with each other and not affecting the continuous phase. The trajectories of particles are predicted by integrating the force balance equation on a particle. The force balance equation in a Lagrangian frame of reference is given by

$\frac{d \vec{v}_{p}}{d t}=a_{D}\left(\vec{v}_{l}-\vec{v}_{p}\right)+\sum_{n=1}^{N} \vec{a}_{n}$

where $a_{D}=\frac{3 \rho_{l} c_{D}\left|\vec{v}_{l}-\vec{v}_{p}\right|}{4 \rho_{p} d_{p}}$ is the absolute value of drag force per unit mass of particle and $C_{D}$ is the drag coefficient. In this equation, $\vec{v}_{p}$ is the velocity vector of the dispersed phase relative to the rotating frame of reference (Motin et al., 2015). The additional acceleration, $\vec{a}_{n}$, can be due to a strong pressure gradient and high density difference between the continuous phase and the discrete phase (see (Motin et al., 2014a; Motin et al., 2014b) for details). Reference (Motin et al., 2015) gives details on numerical simulations and the solution strategies for both the continuous and dispersed phases.

\section{Experimental}

\subsection{Membranes and the feed suspension}

Tubular $\mathrm{TiO}_{2}$ membranes (Filtanium, TAMI Industries) with a $0.14 \mu \mathrm{m}$ nominal pore size were used in all filtration tests in an inside-out flow geometry. Membranes were 250 $\mathrm{mm}$ long with a $10 \mathrm{~mm}$ external diameter, a $6 \mathrm{~mm}$ inner diameter, and an effective filtration area of $\sim 47.1 \mathrm{~cm}^{2}$. Hollow glass microspheres with a density of $0.46 \mathrm{~g} / \mathrm{mL}$ and 
nominal diameters ranging from 5 to $27 \mu \mathrm{m}$ were purchased from Cospheric LCC. Feed suspensions were prepared by adding $1.18 \mathrm{~g}$ of glass microspheres to $4 \mathrm{~L}$ of deionized water and stirring the mixture continuously using a magnetic stirring bar for at least 5 min. A Mastersizer 2000 (Malvern) particle sizer was used to measure the size distribution of microspheres in the suspension. Although the glass microspheres were hollow, the refractive index of glass $(n=1.52)$ was entered as an input for Mastersizer software. Microsphere concentration was determined spectrophotometrically (MultiSpec-1501, Shimadzu) by measuring light transmission at $800 \mathrm{~nm}$.

\subsection{Experimental apparatus and filtration protocol}

Figure 5 shows a diagram of the rotating membrane crossflow filtration apparatus. The feed dispersion of glass microspheres was kept in an open container with continuous magnetic stirring. A diaphragm pump (Hydra-Cell M-03, Wanner Engineering) was used to pump the feed and provide the transmembrane pressure drop, which was maintained at $\sim 0.1 \mathrm{MPa}$ (1 bar) using a back-pressure valve (BP-3, Circle Seal Controls). (The backpressure valve and pump were adjusted periodically during an experiment to maintain a nearly constant retentate flux.) Typical retentate velocities were 0.06 or $0.36 \mathrm{~m} / \mathrm{s}$ at the outlet of the membrane lumen, translating to retentate flow rates of $0.1 \mathrm{~L} / \mathrm{min}$ and $0.61 \mathrm{~L} / \mathrm{min}$, respectively. At the highest permeate flowrate of $0.25 \mathrm{~L} / \mathrm{min}$ (corresponding to the initial permeate flux of $3170 \mathrm{~L}\left(\mathrm{~m}^{2} \cdot \mathrm{h}\right)$ through an unfouled membrane), the feed flow rate was in the $0.35 \mathrm{~L} / \mathrm{min}$ to $0.86 \mathrm{~L} / \mathrm{min}$ range. This corresponds to Reynolds numbers from 1235 to 3028, indicating primarily laminar flow within the membrane channel. Retentate and permeate were returned to the feed tank to keep the liquid volume and the microsphere concentration in the feed as constant as possible. The permeate stream was collected via a ring-shaped basin that accommodated the rotating permeate outlet. The trough had a triangular cross-section so that permeate left the trough through elastic tubing and returned to the feed tank. A pulse dampener (model H1020V, Blacoh Fluid Control) minimized transmembrane pressure fluctuations. Additional details on the experimental apparatus are provided in SM. 


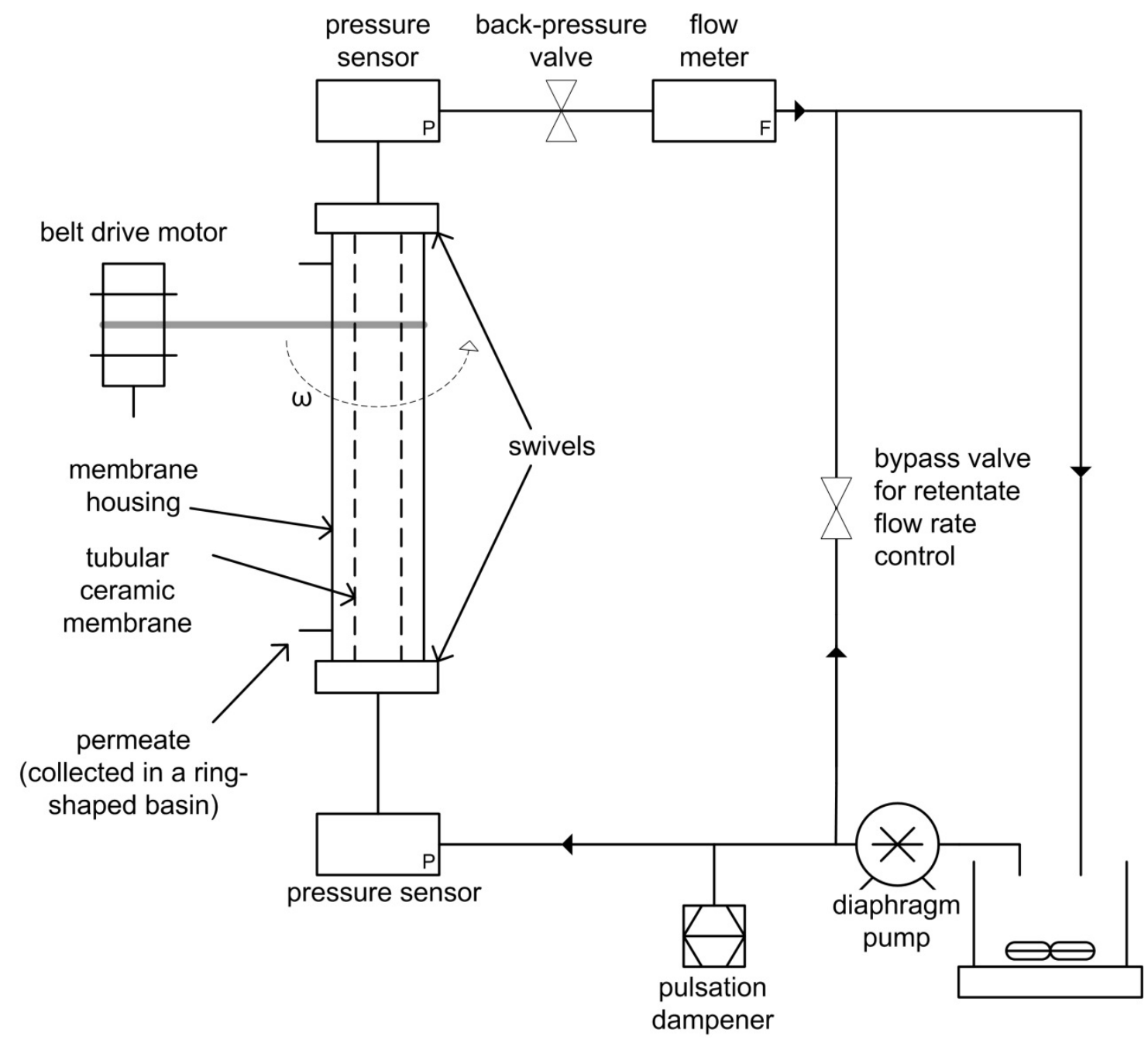

Figure 5: Diagram of the crossflow filtration system with a rotating membrane. Permeate was returned to the feed tank during the filtration (not shown).

\section{Results and Discussion}

This study aims to identify the conditions under which rotating a membrane can decrease fouling by negatively buoyant particles. We employ hollow glass microspheres as model particles because their low density should lead to a high radial velocity toward the center of rotating membranes to reduce fouling even at relatively high permeate fluxes. The sections below describe (1) development of conditions under which particle deposition on membranes occurs and depends on particle size, (2) assessment of particle size in the cake that forms on the membrane surface, (3) modeling of the flow field in the lumen of the rotating membrane, (4) comparison of 
experimental and computational data for the efficiency of particle separation as a function of particle size, (5) assessment of the impact of particle cake erosion by shear forces on the separation efficiency, and (6) application prospects for rotating membranes.

\subsection{Membrane fouling during crossflow filtration}

The first step in this work is identification of crossflow filtration conditions under which fouling occurs and rotation may limit fouling. High crossflow velocities assisted by flotation may drag buoyant particles away from the surface. In an initial experiment with a minimum crossflow rate of $0.36 \mathrm{~m} / \mathrm{s}$ (crossflow rate decreases along the length of the membrane due to permeation) and a transmembrane water flux of $3170 \mathrm{~L} /\left(\mathrm{m}^{2} \cdot \mathrm{h}\right)(\sim 8.8$ $x 10^{-4} \mathrm{~m} / \mathrm{s}$ at the lumen wall), the microsphere concentration in the feed dropped $\sim 35 \%$ over the course of the filtration for both a stationary and a rotating membrane. Part of this drop may stem from breakage of some spheres or loss in the tubing or pump. In contrast, at a relatively low retentate crossflow velocity of $0.06 \mathrm{~m} / \mathrm{s}$, the evolution of the microsphere concentration in the feed suspension, $C_{f}$, during the filtration (Figure 6) suggests that microspheres formed a cake on the stationary membrane and that membrane rotation limits particle deposition. Without membrane rotation, $C_{f}$ dropped $61 \%$ during 30 min of filtration, whereas with a rotating membrane (and the same crossflow velocity), $C_{f}$ decreased only $40 \%$.

Because the microspheres are much larger $(>5 \mu \mathrm{m})$ than the nominal pore size of the membrane $(0.14 \mu \mathrm{m})$, no particles should leave with the permeate stream, and the difference in the decrease in $C_{f}$ for rotating and stationary membrane should result from deposition of microspheres on the membrane surface. However, because the particle diameter is large compared to the membrane pore size, the decline in permeate flux due to the resistance of the cake layer is not significant. The cake mass recovered in our tests ranged from 0.27 to $0.68 \mathrm{~g}$. Assuming a particle diameter of $11.5 \mu \mathrm{m}$ (the 
most abundant particle size in the distribution) and a cake porosity of 0.4 (corresponding to loose random packing of spheres), the Carman-Kozeny equation (Carman, 1937; Kozeny, 1927) gives a value of $\sim 1.2 \times 10^{9} \mathrm{~m}^{-1}$ for the hydraulic resistance of a cake with a mass of $0.68 \mathrm{~g}$. This is almost two orders of magnitude smaller than the hydraulic resistance of the membrane itself $1.13 \times 10^{11} \mathrm{~m}^{-1}$ (calculated based on the pure water flux of $3170 \mathrm{~L} /\left(\mathrm{m}^{2} \cdot \mathrm{h}\right)$ at 1 bar). The thickness of the cake is $0.53 \mathrm{~mm}$ for the highest amount of deposition, indicating that under these conditions cake occupies $\sim 32 \%$ of the membrane channel cross-section.

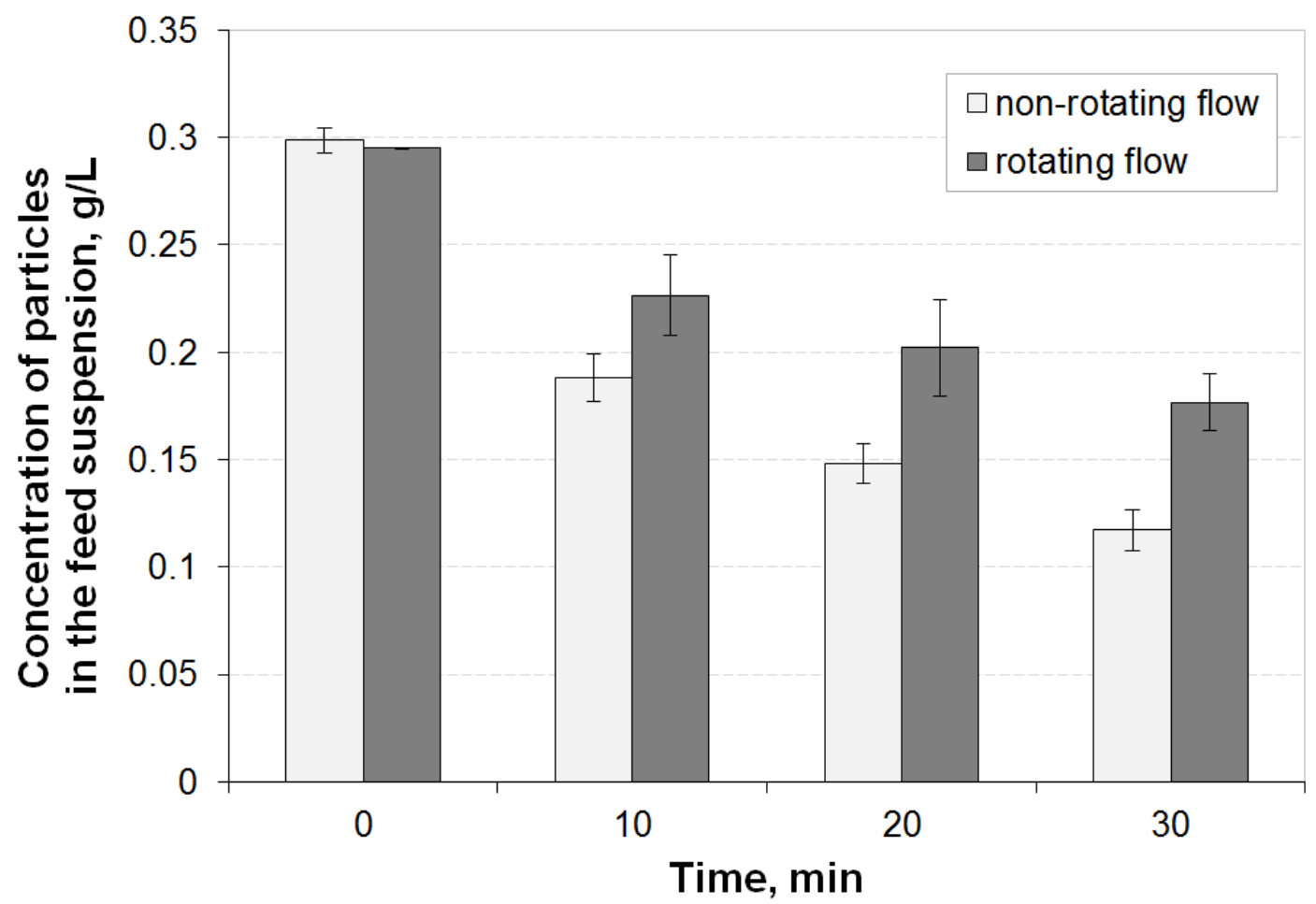

Figure 6: Concentrations of negatively buoyant microspheres in the feed suspension as a function of filtration time in experiments with and without membrane rotation. The crossflow velocity in the membrane channel was $0.06 \mathrm{~m} / \mathrm{s}$ at the lumen outlet. The error bars indicate standard deviations for the concentration of microspheres in the retentate in 3 different filtration tests.

\subsection{Particle size distribution in membrane cakes}

In addition to reducing the mass of particles deposited on membrane walls, membrane rotation should shift the particle size distribution in the cake to lower sizes relative to the 
feed. Thus, this section examines the particle size distribution in the membrane cake both conceptually and experimentally to verify the effect of rotation on particle deposition. Because a particle's radial velocity toward the center of a rotating membrane depends on the square of the particle diameter (see eq. (5)), larger particles are less likely to reach the membrane wall and deposit there. Moreover, particles must have $\vec{v}_{r}$ directed toward the lumen wall to significantly foul the membrane; trajectories of such particles will intercept the surface of the membrane only if the time necessary for a particle to migrate to the surface radially (i.e. in direction opposite to $\vec{e}_{r}$ ) is smaller than $L / v_{p, z}$, where $L$ is the length of the membrane channel.

In any cross-sectional plane normal to the membrane axis, particles that enter the cross-section closer to the membrane wall are more likely to deposit on the wall and contribute to membrane fouling. Thus the size, $d_{p}^{*}$, of the particles that have zero radial velocity near the wall is an approximate lower bound on the size of completely nonfouling particles. In other words, all particles larger than this critical particle diameter $d_{p}^{*}$ should leave the membrane channel with retentate flow because even the smallest of them (the ones with diameter $d_{p}^{*}$ ) entering the channel close to the membrane surface will have $v_{r}=0$ and not move toward the wall.

As defined above, the critical particle diameter, $d_{p}^{*}$, corresponds to particles with $v_{r}=0$ at $r \cong R$. We choose a distance near the wall but not so close that the wall correction to drag is needed. Under these conditions and neglecting inertia, rearrangement of eq. (5) with substitution gives

$j \cong \frac{d_{p}^{* 2}}{18 \mu}\left(\rho_{w}-\rho_{p}\right) \frac{v_{l, \theta}^{2}}{R} \cong \frac{d_{p}^{* 2}}{18 \mu}\left(\rho_{w}-\rho_{p}\right) \frac{\omega^{2} R^{2}}{R} \cong \frac{d_{p}^{* 2}}{18 \mu}\left(\rho_{w}-\rho_{p}\right) \omega^{2} R$

For our experiments, solving equation (25) gives $d_{p}^{*} \cong 17.3 \mu \mathrm{m}$ (Figure 7B) based on the following values: $j=8.8 \times 10^{-4} \mathrm{~m} / \mathrm{s}$ (average pure water flux measured for the 0.14 $\mu \mathrm{m}$ pore size membrane at $\Delta P=0.1 \mathrm{MPa}) ; \mu=1.00 \times 10^{-3} \mathrm{~kg} /(\mathrm{m} \cdot \mathrm{s}) ; \rho_{p}=460 \mathrm{~kg} / \mathrm{m}^{3} ; \rho_{l}=$ 
$998 \mathrm{~kg} / \mathrm{m}^{3} ; \omega=180 \mathrm{~s}^{-1}(1750 \mathrm{rpm}) ; R=3 \times 10^{-3} \mathrm{~m}$. Note that due to inertia, some particles with diameters $>17.3 \mu \mathrm{m}$ will come in contact with the membrane wall.

Using a backflush immediately after filtration, we collected microspheres deposited as a cake on the membrane surface and subsequently determined the size distribution for these particles. Figure 7A shows size distributions of the microspheres in a feed solution as well as in backflush solutions collected after filtrations with stationary and rotating membranes. (In one case, we measured the feed size distribution twice during an experiment, and it did not change significantly.) The gray area denotes the domain of particles larger than $17.3 \mu \mathrm{m}, d_{p}>d_{p}^{*}$. Comparison of the particle size distribution in the feed suspension and in the cake formed on a rotating membrane clearly indicates the diminution of larger particles in the cake, consistent with transport of larger particles away from the wall. (Simulations described below provide a more detailed explanation of the particle distribution in the cake.)

Importantly, the particle size distribution in the cake obtained without membrane rotation did not differ significantly from that in the feed solution, indicating that crossflow alone (i. e. in the absence of rotating flow) did not lead to preferential accumulation of particles of a certain size on the membrane. The crossflow rate $\left(Q_{c f}=1.2 \times 10^{-5}\right.$ to $3.7 \times 10^{-6} \mathrm{~m}^{3} / \mathrm{s}$ along the length of the membrane) was the same for both the rotating and stationary membranes. Thus, these data further confirm that rotation-induced buoyancy forces selectively move larger particles away from the membrane. As noted above (Figure 6), rotation also leads to a decrease in the total mass of particles deposited on the membrane.

\subsection{Flow patterns in rotating membranes}

A more complete understanding of particle collection on membrane walls requires knowledge of the flow pattern within the membrane along with particle trajectories. 
A

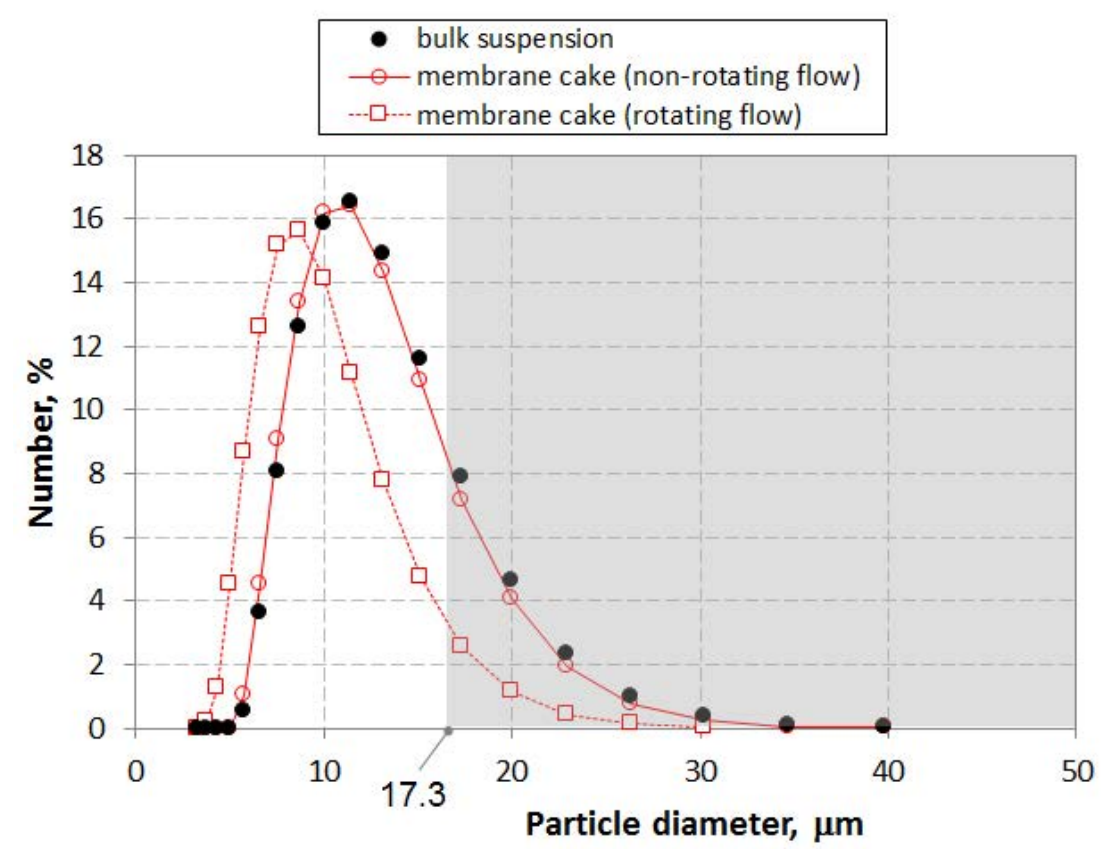

B

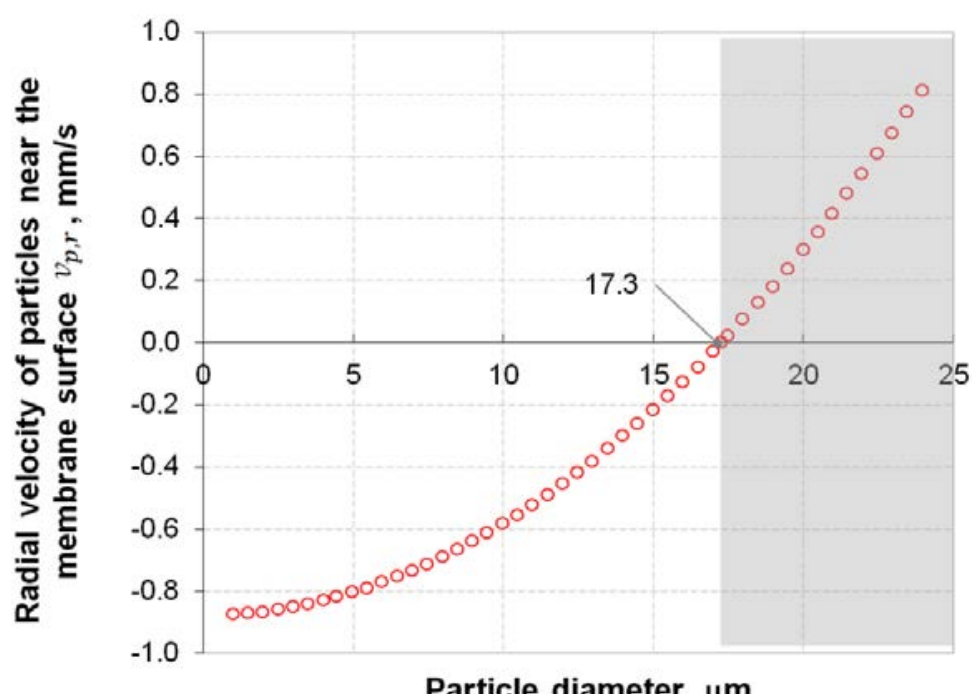

Particle diameter, $\mu \mathrm{m}$

Figure 7: (A) Microsphere size distributions in the bulk of the feed suspension and in membrane cakes collected after filtration experiments with and without membrane rotation. Similar trends appeared in five replicate filtrations. (B) Radial velocity $\left(v_{p, r}\right)$ of particles of different sizes $\left(d_{p}\right)$ at $r \cong R$. All particles larger than $17.3 \mu \mathrm{m}$ should move radially away from the lumen wall. 
The simple force balance approach to determine a critical particle size does not take into account the velocity of particles as they approach the membrane. Thus, this section employs computational fluid dynamics (CFD) to give snapshots of fluid velocity fields and shear stresses in the channel of a rotating tubular membrane. Later sections examine CFD predictions of the number of particles that encounter the membrane wall.

Figure 8A shows the dimensionless axial velocity profile at different cross sections in the axial direction of the rotating crossflow filtration system $(z / L$ is the normalized distance along the membrane axis). The solid line represents the velocity profile of a fully developed Hagen-Poiseuille flow at the inlet of the crossflow filtration. This figure reveals that the axial momentum along the axial direction decreases because the permeate flux is essentially constant along the membrane due to a nearly uniform transmembrane pressure. This results in a regular diminution of axial flow and wall shear stress along the axial direction (see below).

Figure 8B shows the normalized azimuthal velocity profile at different axial locations in the rotating crossflow filtration system. Normalization is with respect to the azimuthal velocity at the lumen wall, i.e. $\left[v_{l, \theta}\right]_{r=R}=\omega R$. The CFD-predicted dependence is different from the idealized linear function shown in Figure 3B. The normalized azimuthal velocity increases with distance along the membrane axis because reduction of the axial velocity downstream (see Figure $8 \mathrm{~A})$ increases the swirl intensity $\left(\omega R / \bar{v}_{l, z}\right)$, indicating that the swirling flow develops in the rotating membrane system. The greater azimuthal velocity downstream yields an increase in the difference of centrifugal force between the two phases, which enhances the probability of the lighter, dispersed phase migrating toward the core. Nevertheless, throughout the membrane lumen, $v_{l, \theta}$ is smaller than $\omega r$, the value for the azimuthal velocity in a completely developed flow. These relatively low values of $v_{l, \theta}$ will lead to greater movement of particles toward the wall and increased fouling. 
A

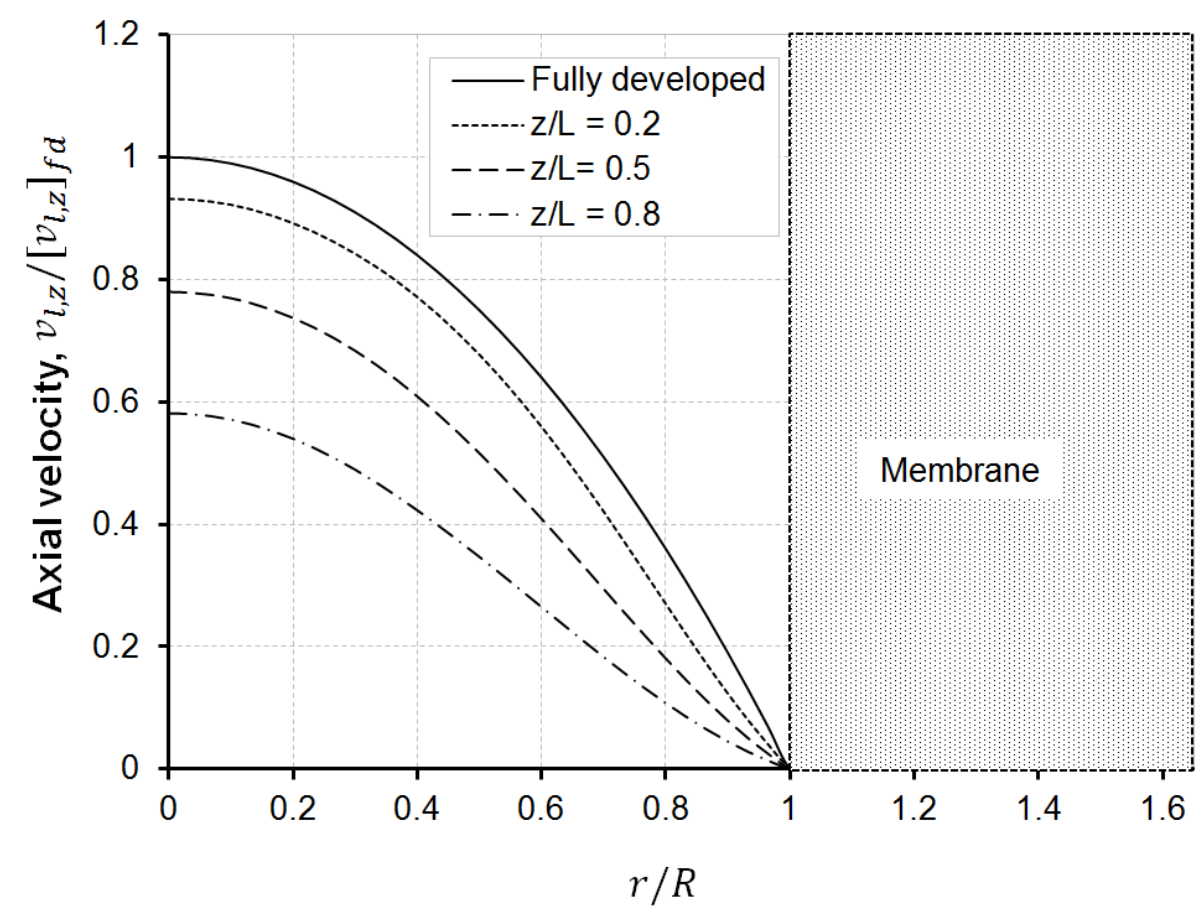

B

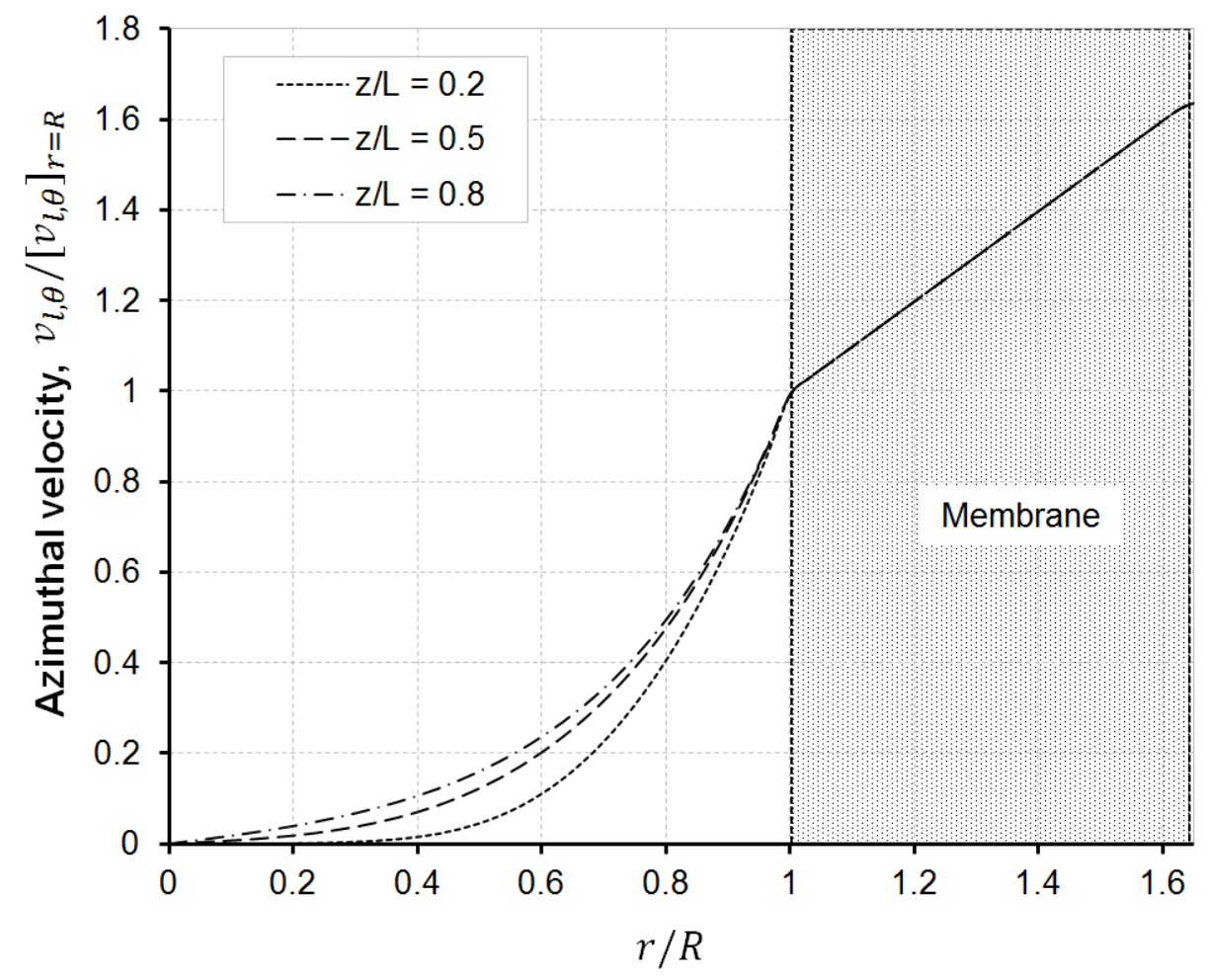




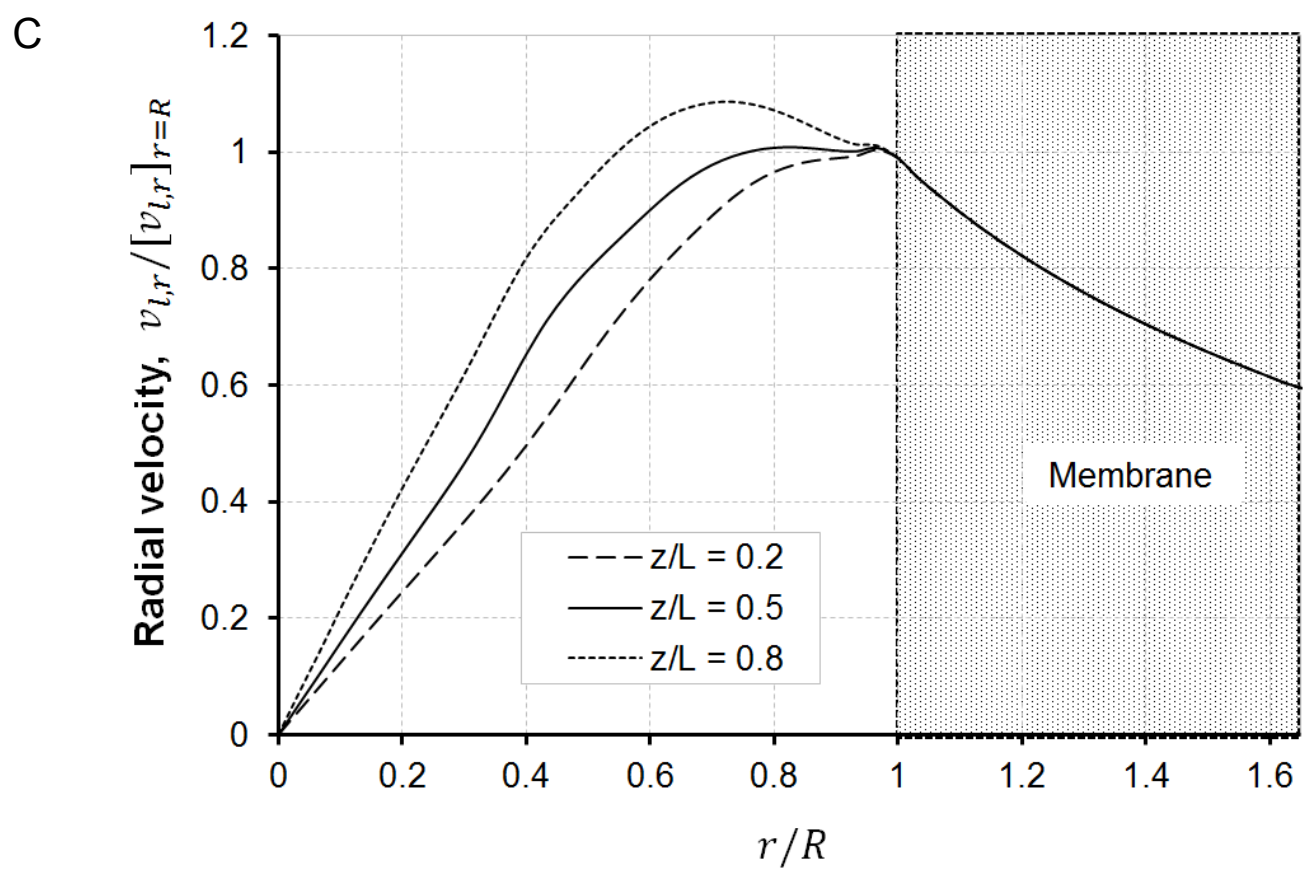

Figure 8: Profiles of $(\mathrm{A})$ axial $\left(v_{l, z}\right),(\mathrm{B})$ azimuthal $\left(v_{l, \theta}\right)$ and $(\mathrm{C})$ radial $\left(v_{l, r}\right)$ velocities at different axial locations along the membrane channel during crossflow filtration. In (A), the solid line is the velocity profile of fully developed Hagen-Poiseuille flow at the inlet. Reynolds and Swirl numbers are: $R e=1270 ; S_{w}=\omega R / \bar{v}_{l, z}=2.6$. $\left[v_{l, z}\right]_{f d}$ denotes the axial component of fluid velocity at the centerline of the fully developed flow.

The fluid radial velocity in the rotating crossflow filtration (Figure $8 \mathrm{C}$ ) gradually increases with distance from the axis of rotation and then peaks near the inner wall of the membrane. The maximum in $v_{r}$ occurs at $r<R$ in part because the radial volumetric flow rate increases on moving from the center toward the wall, but increasing cylindrical areas with greater values of $r$ require lower radial velocities to maintain a given flow rate. Additionally, decreasing axial velocities with increasing $r$ also give rise to less fluid entering the radial flow near the wall. 
Radial velocity also increases in the downstream of the crossflow filtration due to the increase in the centrifugal acceleration (see Figure 8B). This should increase the drag force on the particles and can decrease their migration rate towards the centerline of the flow. However, Figure $8 \mathrm{C}$ also shows that the radial velocity at the membrane wall is uniform along the axial locations. The average transmembrane pressure for these experiments is $0.1 \mathrm{MPa}$, and the total axial pressure loss due to flow should be less than $50 \mathrm{~Pa}$. Thus, the nearly constant transmembrane pressure gives a uniform transmembrane flux throughout the membrane, and the radially outward drag force, $\vec{F}_{j}$, on the particles situated close to the membrane wall is independent of the axial location. Nevertheless, the maximum fluid radial velocities inside the lumen should bring larger than expected particles to the wall with inertia sufficient to foul the membrane. Overall, these flow profiles suggest that the critical particle diameter will underpredict the size of particles that can foul the membrane.

Shear stresses at the membrane wall will affect the extent to which particles are removed from the cake in a crossflow. The shear or traction forces acting on the membrane surface are calculated as $(\vec{\tau})_{r=R}=\tau_{r r} \vec{e}_{r}+\tau_{r z} \vec{e}_{z}+\tau_{r \theta} \vec{e}_{\theta}$. We ignore $\tau_{r r}$ since it is normal to the membrane surface. $\tau_{w z}=\left(\tau_{r z}\right)_{r=R}=\mu_{l}\left[\frac{\partial v_{l, z}}{\partial r}+\frac{\partial v_{l, r}}{\partial z}\right]_{r=R}$ is the average shear component in the axial direction and $\tau_{w \theta}=\left(\tau_{r \theta}\right)_{r=R}=\mu_{l}\left[r \frac{\partial}{\partial r}\left(\frac{v_{l, \theta}}{r}\right)+\right.$ $\left.\frac{1}{r} \frac{\partial v_{l, r}}{\partial \theta}\right]_{r=R}$ is the shear component in the azimuthal direction (Motin et al., 2015).

Figure 9 shows the variation of wall shear stress as a function of the axial distance into the membrane. The axial component of the wall shear stress, $\tau_{w z}$, is $\mu_{l} \dot{\gamma}_{w}$, where the wall shear rate, $\dot{\gamma}_{w}$, is defined by eq. (13). The value of $\mu_{l} \dot{\gamma}_{w}$ computed using eq. (13) is $0.18 \mathrm{~Pa}$ and is a reasonable match to $0.10 \mathrm{~Pa}$, which is the CFD-predicted value of $\tau_{w z}$ averaged over membrane length (Figure 9). Both the axial and azimuthal components of wall shear stress gradually decrease along the axial direction. However, because of 
the high angular speed of membrane, the azimuthal component $\left(\tau_{w \theta}\right)$ is much higher than the axial component $\left(\tau_{w z}\right)$. The increased resultant wall shear stress due to the higher swirling flow should help to dislodge particles from the membrane surface and allow axial crossflow to sweep them away and reduce their accumulation.

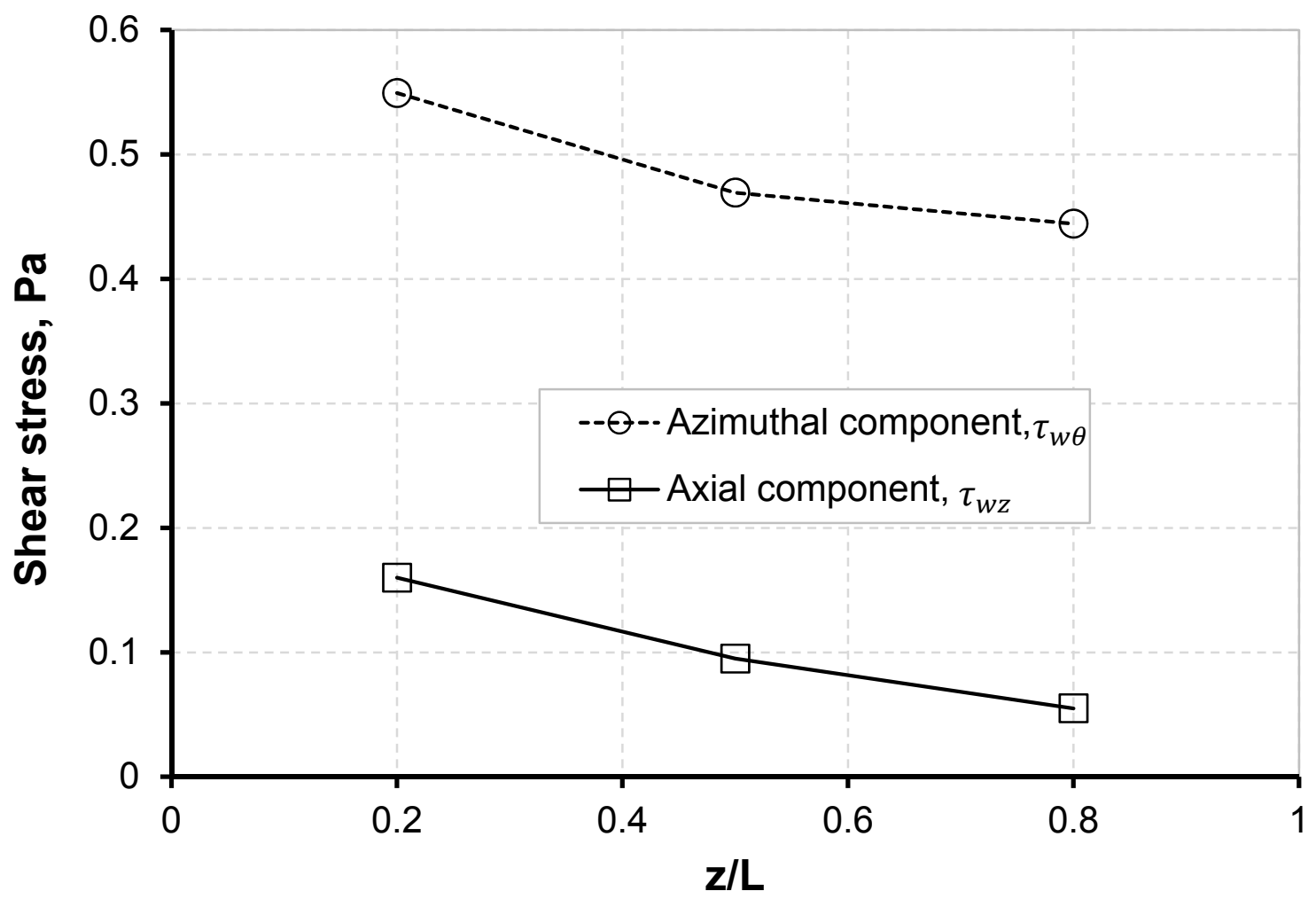

Figure 9: Axial and azimuthal components of shear stress along the inner wall of membrane. 


\subsection{Separation efficiency}

Using the computed fluid flow pattern and particle trajectories in the rotating membrane, we estimated the fraction of particles of a given size that reach the membrane wall. Assuming all of these particles remain on the wall, this fraction corresponds to $1-G_{T}^{\text {no erosion. }}$. Based on this value and the flow ratio, $R_{f}$, of 0.28 we determined

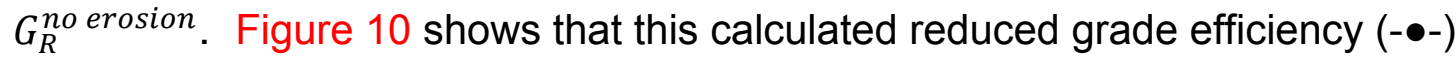
increases with increasing particle size, as expected. Notably, at the critical particle size of $17.3 \mu \mathrm{m}, G_{R}^{\text {no erosion }}$ is approximately 0.5 , consistent with inertial effects decreasing $G_{R}$ compared to the value predicted with a simple force balance.

Experimental determination of $G_{R}$ for different particle sizes requires size distributions in the cake and the initial feed along with values for the mass of the cake and $R_{f}$. To account for the change in the feed size distribution throughout the experiments, we employ eq. (26), where $G_{T}$ is the total grade efficiency for a given particle size range, $V_{f}$ is the volume of the feed solution (nearly constant because of recycling), $Q_{f}$ is the feed volumetric flow rate, $t$ is the filtration time, $N_{i, w a l l}$ is the number of particles of the given size range in the wall, and $N_{i, f}(t=0)$ is the number of particles of that size range in the feed at the beginning of the filtration. (See SM for a derivation of this equation.)

$G_{T}=1-\frac{V_{f}}{Q_{f} t} \ln \left[1-\frac{N_{i, w a l l}}{N_{i, f}(t=0)}\right]$

Subsequently, we use eq. (18a) to calculate $G_{R}$.

As Figure 10 shows, the experimental data (-o-) show higher values of $G_{R}$ than the CFD-predicted values of $G_{R}^{\text {no erosion }}(-\bullet-)$. This likely reflects the computational assumption that all particles that encounter the membrane wall remain in the cake. In reality, shear forces will remove particles from the wall, and sticking is a complicated function of surface energies, particle size and hydrodynamics (see below). 


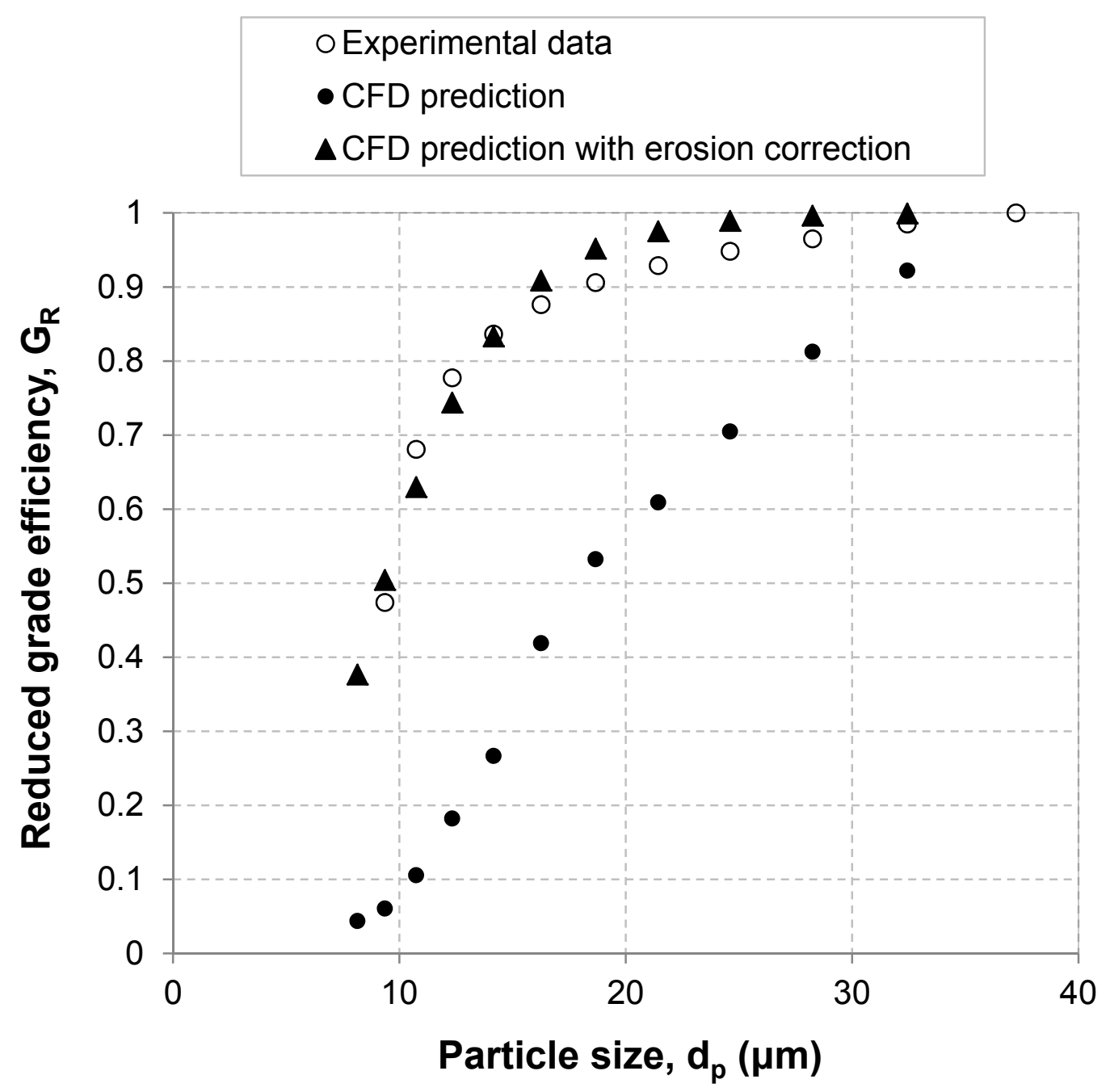

Figure 10: Effect of particle size on the experimental (-०-) and CFD-predicted $\left(G_{R}^{\text {no erosion }},-\bullet-\right)$ values of reduced grade efficiency for crossflow filtration with rotation at $1725 \mathrm{rpm}$. The figure also shows the CFD values corrected for erosion (- $\left.\mathbf{\Delta -}, G_{R}^{\text {erosion }}\right)$. Feed flow rate $=0.36$ $\mathrm{L} / \mathrm{min}$, retentate flow rate $=0.1 \mathrm{~L} / \mathrm{min}$, permeate flux $=3200 \mathrm{~L} /\left(\mathrm{m}^{2} . \mathrm{hr}\right)$, and transmembrane pressure is $0.1 \mathrm{MPa}$.

Perhaps the most important conclusion from Figure 10 is that nearly $100 \%$ separation occurs for particles with diameters larger than $\sim 35 \mu \mathrm{m}$. In other words, for the given operating conditions, particles larger than $\sim 35 \mu \mathrm{m}$ should not significantly foul the 
membrane. For small particles ( $d_{p}$ less than $\sim 5 \mu \mathrm{m}$ ) the rotation-based separation under the given operating condition is ineffective; most of these particles will deposit on the membrane and block membrane pores or form a membrane cake. Rotationally induced buoyancy forces on these particles are essentially negligible.

\subsection{Effect of erosion on the separation efficiency}

Erosion of the cake due to the crossflow drag on the particles will increase $G_{R}$ compared to CFD calculations that assume all particles encountering the lumen wall remain there. Modifying the CFD data by taking into account erosion (eqs. (15), (16), (20b), (22)) using $\delta_{e}$ as the fitting parameter in the expression for the erosion parameter, $\kappa$ (eq. $17 a)$, yields a much improved fit (-o- vs - $\Delta-$ ) to the experimental data and a $\delta_{e}$ value of $5.7 \mu \mathrm{m}$. This value corresponds to the cut-diameter; that is to say particles with diameters $<\delta_{e}$ should not erode with the crossflow. Figure S1 (see SM) gives values of $\kappa$ as a function of particle size.

We employed the moment balance on a single particle (eq. (14)) for our experimental conditions to assess whether a cut-diameter of $5.7 \mu \mathrm{m}$ is reasonable. Assuming that the angle of repose $\theta$ is $35.3^{\circ}$ (the value for hexagonal packing of spheres adapted by Chang et al. (Chang et al., 1995)), the moment balance yields a cut-diameter value of $58.5 \mu \mathrm{m}$, and the experimental cut-diameter of $5.7 \mu \mathrm{m}$ (see previous section) requires $\theta \cong 13.5^{\circ}$. The lower angle is more consistent with values of $\theta \cong 6.3^{\circ}$ determined by Lu and Ju for microfiltration of a $\mathrm{CaCO}_{3}$ slurry (Lu and Ju, 1989) and $\theta \cong 17^{\circ}$ found by Vyas et al. for microfiltration of lactalbumin particles (Vyas et al., 2001). The combination of $\theta \cong 13.5^{\circ}$ and $\delta_{e} \cong 5.7 \mu \mathrm{m}$ explains most of the discrepancy between experimental data and the CFD prediction (Figure 10). Indeed, extrapolating experimental data to the point of zero reduced grade efficiency gives the particle size value between 5 and $10 \mu \mathrm{m}$. This size is smaller the critical particle diameter $d_{p}^{*}=17.3$ $\mu \mathrm{m}$. Thus, in our experimental system erosion likely plays an important role in removing particle in the 5 to $17 \mu \mathrm{m}$ size range. 
The proposed effect of erosion seems inconsistent with the particle size distribution (Figure 7) that indicates that crossflow in the absence of membrane rotation does not lead to preferential accumulation of smaller particles in the membrane cake. (Erosion should selectively remove large particles from the cake.) However, this apparent contradiction can be explained by assuming that the azimuthal shear stress dislodges particles. Indeed, $\tau_{w \theta}$ is $\sim 5$ times higher than $\tau_{w z}(0.5 \mathrm{~Pa}$ versus $0.1 \mathrm{~Pa}$, see Figure 9$)$. The assumption that $\tau_{w \theta}$ and not $\tau_{w z}$ removes particles from the cake predicts that the cut-diameter $\delta_{e} \cong 5.7 \mu \mathrm{m}$ corresponds to the angle of repose $\theta \cong 33.5^{\circ}$ (eqs (14), (17a), (20b), (22)), close to the angle corresponding to hexagonal packing $\left(35.3^{\circ}\right)$. While $\tau_{w \theta}$ does not direct sheared particles axially toward the exit from the membrane channel, it should facilitate entrainment of particles in the crossflow. Under this scenario, crossflow will only erode the cake in the presence of rotation, which is consistent with our experimental data (Figure 7).

The data in Figure 10 represent only one data set, and Figure S2 (see SM) shows data for a replicate experiment. Although the particle size distributions in these experiments are highly reproducible, accurately collecting and drying the cake is more difficult. The cake mass directly affects the value of $G_{T}$ and, hence, $G_{R}$. Despite this uncertainty, the values of $G_{R}$ as a function of particles size are reasonably similar for the two data sets. Fitting the second data set leads to a $\delta_{e}$ value of $6.9 \mu \mathrm{m}$, which is slightly larger than the value of $5.7 \mu \mathrm{m}$ given above. The corresponding value of $\theta$ (angle of repose) is $14.1^{\circ}$ for the second data set compared to $13.5^{\circ}$ for the data in Figure 10. If we assume that the azimuthal component of the shear stress dislodges particles, then $\delta_{e}=6.9 \mu \mathrm{m}$ translates into $\theta \cong 34.8^{\circ}$ compared to $33.5^{\circ}$ for the first data set. Recall that $\theta$ for hexagonal packing is $35.3^{\circ}$. Both data sets give reasonable values of the cut-diameter and angle of repose.

\subsection{Prospects for rotating membranes}

Despite fast rotation $(\omega=1725 \mathrm{rpm})$ and a large density difference $(\Delta \rho \approx 0.54 \mathrm{~g} / \mathrm{mL}$ ) between continuous and dispersed phases, rotation-induced reductions in particle 
deposition on the membrane surface are limited. At the permeate flux of $8.8 \times 10^{-4} \mathrm{~m} / \mathrm{s}$ (3170 LMH), rotation significantly reduces cake formation only for particles larger than 8 $\mu \mathrm{m}$. The value of $\omega$ required for particle separation depends on $j$ and $\Delta \rho$, but, for a given particle size, this dependence is relatively weak: $\omega \sim \frac{1}{d_{p}} \sqrt{\frac{j}{R \Delta \rho}}$ (see eq. (5)). However, for oil emulsions, a $\Delta \rho$ value of only $0.12 \mathrm{~g} / \mathrm{mL}$ or less could easily require more than doubling the rotation rate compared to what we employed in this study (1725 rpm), unless the oil droplets coalesce near the membrane surface. Larger membranes with increased radii would require lower rotation rates, but rotation of membranes at large angular velocities may be impractical, especially when the membrane diameter is large so high torques require more power. Operating membrane at lower fluxes will decrease the required rotation rate, but of course this will decrease productivity. Another disadvantage of rotating membranes is that the feed should be free of particles that are denser than the continuous phase because they would be driven by the centrifugal force to the membrane surface and would foul the membrane.

Nevertheless, membranes are attractive for treatment of oil-in-water emulsions because they remove small droplets with the cutoff size controlled by the choice of membrane pore size. Oil-in-water dispersions such as produced water may contain a large fraction (40\% (TORR Canada, 2007)) of oil in droplets that are too small for removal with conventional oil-water separation technologies such as hydrocyclones and dissolved air flotation (Frankiewicz, 2001). A microfiltration membrane can remove such droplets albeit at the expense of membrane fouling. In the proposed rotational system, centripetal forces will limit this drawback for droplets larger than a given size. The DCF technology might prove useful for removing small droplets in a one-pass treatment. Although a treatment train may remove small droplets (e.g. secondary pretreatment by a hydrocyclone or dissolved air flotation followed by conventional microfiltration with a stationary membrane), a rotating membrane without the secondary pretreatment step may require less space and time. 


\section{Conclusions}

This proof-of-concept study describes crossflow filtration with a rotating membrane where hollow glass microspheres ( $\rho \approx 0.46 \mathrm{~g} / \mathrm{mL}$ ) serve as a model system for evaluating the effects of membrane rotation on the accumulation of particles at the membrane surface. At low crossflow velocities, rotating the membrane leads to less loss of microspheres from the feed solution than filtration with a stationary membrane. Moreover, the size distribution of microspheres recovered from the particle cake formed on the membrane shifts to lower diameters only for filtrations performed with membrane rotation. These effects stem from the rotation-induced centripetal force that preferentially moves larger particles away from the membrane. Shear forces should also preferentially dislodge large particles ( $>5$ to $10 \mu \mathrm{m}$ ) from the cake. A force balance on a microsphere demonstrates that rotation and crossflow should greatly limit access to the membrane surface for particles with diameters $>17 \mu \mathrm{m}$. Computational fluid dynamics simulations show that the rotating flow does not fully develop in the membrane, and inertial effects may lead to deposition of particles even when their diameter exceeds $17 \mu \mathrm{m}$. At the rotation rate in this work (1725 rpm), particles with diameters $>35 \mu \mathrm{m}$ are essentially completely excluded from the membrane, whereas rotation has minimal effect on particles with diameters $<8 \mu \mathrm{m}$. A similar effect with oil droplets in water will require higher flow rates or lower fluxes, but a rotating membrane may avoid a pretreatment step to remove large oil droplets.

\section{Acknowledgements}

This research was supported in part by the Environmental Protection Agency Science to Achieve Results Grant RC-83518301, National Science Foundation Grant IIA-1243433, and Michigan State University Foundation Grant 71-1624. We thank Thomas Davis for his help with assembling the rotating membrane system. 


\section{Nomenclature}

$\delta_{e} \quad$ cut-diameter (due to erosion, particles larger than $\delta_{e}$ are prevented from depositing on the membrane surface)

$\dot{\gamma}_{w} \quad$ shear rate at the lumen wall

$\theta \quad$ angle of repose of a particle

$\kappa \quad$ erosion parameter

$\mu_{l} \quad$ viscosity of the dispersion phase (water)

$\rho_{l} \quad$ density of the dispersion phase (water)

$\rho_{p} \quad$ density of the particles

$\phi \quad$ wall correction factor to the drag force on a particle

$\omega \quad$ angular velocity

$a_{D} \quad$ absolute value of drag force per unit mass of particle

$C_{f} \quad$ concentration of microspheres in the feed suspension

$C_{D} \quad$ drag coefficient

$C_{w} \quad$ concentration of microspheres at the membrane wall

$d_{p} \quad$ diameter of the particle

$d_{p}^{*} \quad$ critical particle diameter (due to the rotation-induced centripetal force, particles larger than $d_{p}^{*}$ are prevented from reaching the membrane surface)

$\vec{e}_{r}, \vec{e}_{z}$, and $\vec{e}_{\theta}$ unit vectors in the radial, vertical, and azimuthal directions, respectively $\vec{F}_{c} \quad$ centripetal force on particles

$\vec{F}_{D} \quad$ total drag force on a particle

$\vec{F}_{J} \quad$ drag force on a particle due to permeate flux

$G_{R} \quad$ reduced grade efficiency

$G_{T} \quad$ total grade efficiency

$j \quad$ permeate flux

$\dot{m} \quad$ mass flow rate of particles 


$\begin{array}{ll}N_{i, f} & \text { number of particles of a given size in the feed } \\ N_{i, w a l l} & \text { number of particles of a given size in the cake at the end of the filtration } \\ R & \text { radius of membrane channel } \\ R e & \text { Reynolds number } \\ R e_{p} & \text { particle Reynolds number } \\ r & \text { distance from the center of the membrane channel } \\ Q_{c f} & \text { crossflow rate } \\ Q_{f} & \text { volumetric feed flow rate } \\ Q_{r} & \text { volumetric retentate flow rate } \\ Q_{p} & \text { volumetric permeate flow rate } \\ S_{w} & \text { Swirl number } \\ t & \text { total filtration time } \\ \vec{v}_{c f} & \text { crossflow velocity } \\ \vec{v}_{l} & \text { velocity of the dispersion (liquid) phase } \\ \vec{v}_{l i f t} & \text { velocity of particle due to inertial lift } \\ \vec{v}_{p} & \text { velocity of particle } \\ v_{\theta} \vec{e}_{\theta} & \text { azimuthal velocity } \\ v_{r} \vec{e}_{r} & \text { radial velocity } \\ v_{z} \vec{e}_{z} & \text { axial velocity } \\ V_{f} & \text { volume of the feed suspension } \\ \end{array}$




\section{List of Figures}

Figure 1 Diagram of crossflow filtration of a particle suspension through a rotating, solvent-permeable membrane.

Figure 2 Force balance in a radial direction on a suspended negatively buoyant particle in a tubular channel with impervious walls $(A)$ and in tubular membranes in the presence of permeate flow (B, C). As the rate of permeation through the membrane increases beyond a critical value, the direction of particle movement reverses from centripetal $(B)$ to centrifugal $(C)$.

Figure 3 Conceptual radial distribution of azimuthal velocities, $v_{l, \theta}(r)$, for rotating flow inside $(A)$ a stationary membrane and $(B)$ a rotating membrane. In the former case, flow rotation stems from introducing the feed into the membrane channel under an angle. In the latter case, the flow enters the membrane channel co-axially with the channel, and momentum transfer from the rotating wall will rotate the fluid. Figure $8 \mathrm{c}$ shows more realistic velocity profiles for the rotating membrane, as the fluid does not rotate as a block.

Figure 4 Hydrodynamic forces acting on a buoyant microsphere positioned on a deposit of similar particles at the surface of a rotating crossflow filtration membrane. See the text for the definitions of forces.

Figure 5 Diagram of the crossflow filtration system with a rotating membrane. Permeate was returned to the feed tank during the filtration (not shown).

Figure 6 Concentrations of negatively buoyant microspheres in the feed suspension as a function of filtration time in experiments with and without membrane rotation. The crossflow velocity in the membrane channel was $0.06 \mathrm{~m} / \mathrm{s}$ at the lumen outlet. The error bars indicate standard deviations for the concentration of microspheres in the retentate in 3 different filtration tests.

$\begin{array}{ll}\text { Figure } 7 & \text { (A) Microsphere size distributions in the bulk of the feed }\end{array}$ suspension and in membrane cakes collected after filtration experiments with and without membrane rotation. Similar trends appeared in five replicate filtrations. (B) Radial velocity $\left(v_{p, r}\right)$ of particles of different sizes $\left(d_{p}\right)$ at $r \cong R$. All particles larger than $17.3 \mu \mathrm{m}$ should move radially away from the lumen wall. 
Figure 8 Profiles of $(\mathrm{A})$ axial $\left(v_{l, z}\right),(\mathrm{B})$ azimuthal $\left(v_{l, \theta}\right)$ and $(\mathrm{C})$ radial $\left(v_{l, r}\right)$ velocities at different axial locations along the membrane channel during crossflow filtration. In (A), the solid line is the velocity profile of fully developed Hagen-Poiseuille flow at the inlet. Reynolds and Swirl numbers are: $R e=1270 ; S_{w}=\omega R / \bar{v}_{l, z}=2.6$. $\left[v_{z}\right]_{f d}$ denotes the axial component of fluid velocity at the centerline of the fully developed flow.

Figure 9 Axial and azimuthal components of shear stress along the inner wall of membrane.

Figure 10 Effect of particle size on the experimental and CFD-predicted $\left(G_{R}^{\text {no erosion }}\right)$ reduced grade efficiency for the crossflow filtration with rotation at $1725 \mathrm{rpm}$. The figure also shows the CFD value corrected for erosion. Feed flow rate $=0.36 \mathrm{~L} / \mathrm{min}$, retentate flow rate $=0.1 \mathrm{~L} / \mathrm{min}$, permeate flux $=3200 \mathrm{~L} /\left(\mathrm{m}^{2} . \mathrm{hr}\right)$, and transmembrane pressure is $0.1 \mathrm{MPa}$. 


\section{References}

Aubert, M.C., Elluard, M.P., Barnier, H., 1993. Shear stress induced erosion of filtration cake studied by a flat rotating disk method: Determination of the critical shear stress erosion. J. Membr. Sci. 84, 229-240.

Beaudoin, G., Jaffrin, M.Y., 1989. Plasma filtration in Couette flow membrane devices. Artificial Organs 13, 43-51.

Beier, S.P., Guerra, M., Garde, A., Jonsson, G., 2006. Dynamic microfiltration with a vibrating hollow fiber membrane module: Filtration of yeast suspensions. J. Membr. Sci. 281, 281-287.

Belfort, G., Mikulasek, P., Pimbley, J.M., Chung, K.Y., 1993a. Diagnosis of membrane fouling using a rotating annular filter. 2. Dilute particle suspensions of known particle size. J. Membr. Sci. 77, 23-39.

Belfort, G., Pimbley, J.M., Greiner, A., Chung, K.Y., 1993b. Diagnosis of membrane fouling using a rotating annular filter. 1. Cell culture media. J. Membr. Sci. 77, 1-22.

Benard, A., Bruening, M., Gorobets, A., Hogg, S., Ji, P., Motin, A., Shan, W., Starr, B., Yang, Z., 2014. Separation of oil-water dispersions using rotating flow and charged hydrophilic membranes, 2014 Produced Water Society seminar, Houston, TX.

Bendick, J., Reed, B., Morrow, P., Carole, T., 2014. Effect of backpulsing and continuous surface cleaning on high-shear rotary membrane system permeate flux performance for naval shipboard wastewaters. J. Environ. Eng. ASCE 140, 0401400404014009.

Bhattacharjee, C., Bhattacharya, P.K., 2006. Ultrafiltration of black liquor using rotating disk membrane module. Separ. Purif. Technol. 49, 281-290.

Bouzerar, R., Jaffrin, M.Y., Lefevre, A., Paullier, P., 2000. Concentration of ferric hydroxide suspensions in saline medium by dynamic cross-flow filtration. J. Membr. Sci. $165,111-123$.

Brou, A., Ding, L., Boulnois, P., Jaffrin, M.Y., 2002. Dynamic microfiltration of yeast suspensions using rotating disks equipped with vanes. J. Membr. Sci. 197, 269-282.

Carman, P.C., 1937. Fluid flow through granular beds. Trans. Inst. Chem. Eng. 15, 150166.

Carstensen, F., Apel, A., Wessling, M., 2012. In situ product recovery: Submerged membranes vs. external loop membranes. J. Membr. Sci. 394-395, 1-36.

Chang, D.-J., Hsu, F.-C., Hwang, S.-J., 1995. Steady-state permeate flux of cross-flow microfiltration. J. Membr. Sci. 98, 97-106. 
Choi, C.K., Park, J.Y., Park, W.C., Kim, J.J., 1999. A study on dynamic separation of silica slurry using a rotating membrane filter: 2 . Modelling of cake formation. J. Membr. Sci. 157, 177-187.

Davis, R.H., Birdsell, S.A., 1987. Hydrodynamic model and experiments for crossflow microfiltration. Chem. Eng. Commun. 49, 217-234.

Ding, L.-H., Akoum, O., Abraham, A., Jaffrin, M.Y., 2003. High shear skim milk ultrafiltration using rotating disk filtration systems. AIChE J. 49, 2433-2441.

Ding, L.H., Jaffrin, M.Y., Mellal, M., He, G., 2006. Investigation of performances of a multishaft disk (MSD) system with overlapping ceramic membranes in microfiltration of mineral suspensions. J. Membr. Sci. 276, 232-240.

Dolecek, P., Mikulasek, P., Belfort, G., 1995 The performance of a rotating filter 1. Theoretical analysis of the flow in an annulus with a rotating inner porous wall. J. Membr. Sci. 99, 241-248.

El Rayess, Y., Albasi, C., Bacchin, P., Taillandier, P., Raynal, J., Mietton-Peuchot, M., Devatine, A., 2011. Cross-flow microfiltration applied to oenology: A review. J. Membr. Sci. 382, 1-19.

Engler, J., Wiesner, M.R., 2000. Particle fouling of a rotating membrane disk. Water Res. 34, 557-565.

Espina, V.S., Jaffrin, M.Y., Frappart, M., Ding, L.-H., 2008. Separation of casein micelles from whey proteins by high shear microfiltration of skim milk using rotating ceramic membranes and organic membranes in a rotating disk module. J. Membr. Sci. $325,872-879$.

Frankiewicz, T., 2001. Understanding the fundamentals of water treatment: the Dirty Dozen - 12 common causes of poor water quality, 11th Produced Water Seminar, Houston, TX.

Frappart, M., Akoum, O., Ding, L.H., Jaffrin, M.Y., 2006. Treatment of dairy process waters modelled by diluted milk using dynamic nanofiltration with a rotating disk module. J. Membr. Sci. 282 465-472.

Frenander, U., Jönsson, A.S., 1996. Cell harvesting by cross-flow microfiltration using a shear-enhanced module. Biotechnol. Bioeng. 52, 397-403.

Genkin, G., Waite, T.D., Fane, A.G., Chang, S., 2006. The effect of vibration and coagulant addition on the filtration performance of submerged hollow fibre membranes. J. Membr. Sci. 281, 726-734.

Hallstrom, B., Lopez-Leiva, M., 1978. Description of a rotating ultrafiltraton module. Desalination 24, 273-279. 
He, G., Ding, L.H., Paullier, P., Jaffrin, M.Y., 2007. Experimental study of a dynamic filtration system with overlapping ceramic membranes and non-permeating disks rotating at independent speeds. J. Membr. Sci. 300, 63-70.

Holeschovsky, U.B., Cooney, C.L., 1991. Quantitative description of ultrafiltration in a rotating filtration device. AIChE J. 37, 1219-1226.

Jaffrin, M.Y., 2008. Dynamic shear-enhanced membrane filtration: A review of rotating disks, rotating membranes and vibrating systems. J. Membr. Sci. 324, 7-25.

Jaffrin, M.Y., 2012. Hydrodynamic techniques to enhance membrane filtration. Ann. Rev. Fluid Mech. 44, 77-96.

Kaplan, A.A., Halley, S.E., 1990. Plasma exchange with a rotating filter. Kidney Int 38, 160-166.

Kozeny, J., 1927. Ueber kapillare Leitung des Wassers im Boden. Sitzungsber Akad. Wiss., Wien 136, 271-306.

Kroner, K.H., Nissinen, V., 1988. Dynamic filtration of microbial suspensions using an axially rotating filter. J. Membr. Sci. 36, 85-100.

Kroner, K.H., Nissinen, V., Ziegler, H., 1987. Improved dynamic filtration of microbial suspensions. Bio/Technology 5, 921-926.

Le-Clech, P., Chen, V., Fane, T.A.G., 2006. Fouling in membrane bioreactors used in wastewater treatment. J. Membr. Sci. 284, 17-53.

Lee, H., He, F., Song, L., Gilron, J., Sirkar, K.K., 2011. Desalination with a cascade of cross-flow hollow fiber membrane distillation devices integrated with a heat exchanger. AIChE J. 57, 1780-1795.

Lee, S.S., Burt, A., Russotti, G., Buckland, B., 1995. Microfiltration of recombinant yeast cells using disk dynamic filtration system. Biotechnol. Bioeng. 48, 386-400.

Leonard, E.G., Vassiliefl, C.S., 1984. The deposition of rejected matter in membrane separation processes. Chem. Eng. Commun. 30, 209-217.

Li, L., Ding, L., Tu, Z., Wan, Y., Clausse, D., Lanoisellé, J.-L., 2009. Recovery of linseed oil dispersed within an oil-in-water emulsion using hydrophilic membrane by rotating disk filtration system. J. Membr. Sci. 342, 70-79.

Liu, L., Gao, B., Liu, J., Yang, F., 2012. Rotating a helical membrane for turbulence enhancement and fouling reduction. Chem. Eng. J. 181- 182 486- 493.

Lu, W.-M., Ju, S.-C., 1989. Selective particle deposition in crossflow filtration. Separ. Sci. Technol. 24, 517-540. 
Mänttäri, M., Viitikko, K., Nyström, M., 2006. Nanofiltration of biologically treated effluents from the pulp and paper industry. J. Membr. Sci. 272, 152-160.

Mikulášek, P., Doleček, P., 1994. Use of a rotating filter to enhance ceramic membrane filtration performance of latex dispersions. Separ. Sci. Technol. 29, 1943 - 1956.

Motin, A., Gaustad, M.D., Tarabara, V.V., Benard, A., 2013. Simulations and performance of the crossflow filtration hydrocyclone (CFFH) for oil-water separation. Paper No. FEDSM2013-16195, ASME 2013 Fluids Engineering Division Summer Meeting, Incline Village, NV, p. V01CT18A004.

Motin, A., Tarabara, V.V., Benard, A., 2014a. Numerical study of internal flow structure within hydrocyclones with parabolic and hyperbolic swirl chambers, International Mechanical Engineering Congress and Exposition, Montreal, Canada.

Motin, A., Tarabara, V.V., Benard, A., 2015. Numerical investigation of the performance and hydrodynamics of a rotating tubular membrane used for liquid-liquid separation. J. Membr. Sci. 473, 245-255.

Motin, A., Tarabara, V.V., Bénard, A., 2014b. CFD study of hydrodynamics and separation performance of a novel crossflow filtration hydrocyclone (CFFH). Paper No. FEDSM2014-21289, ASME 2014 4th Joint US-European Fluids Engineering Division Summer Meeting. , Chicago, IL, p. V01CT16A008.

Murase, T., Pradistsuwana, C., Iritani, E., Kano, K., 1991. Dynamic microfiltration of dilute slurries with a rotating ceramic membrane. J. Membr. Sci. 62, 187-199.

Nie, C., Ma, L., Xia, Y., He, C., Deng, J., Wang, L., Cheng, C., Sun, S., Zhao, C., 2015. Novel heparin-mimicking polymer brush grafted carbon nanotube/PES composite membranes for safe and efficient blood purification. J. Membr. Sci. 475, 455-468.

O'Neill, M.E., 1968. A sphere in contact with a plane wall in a slow linear shear flow. Chem. Eng. Sci. 23.

Ochirkhuyag, B., Takamasa Mori, T., Katsuoka, T., Satone, H., Tsubaki, J., Choi, H., Sugimoto, T., 2008. Development of a high-performance cake-less continuous filtration system. Chem. Eng. Sci. 63, 5274 -- 5282.

Ould-Dris, A., Jaffrin, M.Y., Si-Hassen, D., Neggaz, Y., 2000. Analysis of cake build-up and removal in cross-flow microfiltration of $\mathrm{CaCO} 3$ suspensions under varying conditions. J. Membr. Sci. 175, 267-283.

Park, J.Y., Choi, C.K., Kim, J.J., 1994. A study on dynamic separation of silica slurry using a rotating membrane filter 1. Experiments and filtrate fluxes. J. Membr. Sci. 97 263-273.

Reed, B.E., Lin, W., Viadero Jr., R., Young, J., 1997. Treatment of oily wates using high shear rotary ultrafiltration. J. Environ. Eng. ASCE 123, 1234-1242. 
Rock, G., Tittley, P., McCombie, N., 1986. Plasma collection using an automated membrane device. Transfusion 26, 269-271.

Saffman, P.G., 1956. On the motion of small spheroidal particles in a viscous liquid. J. Fluid Mech. 1.

Sarkar, D., Datta, D., Sen, D., Bhattacharjee, C., 2011. Simulation of continuous stirred rotating disk-membrane module: An approach based on surface renewal theory. Chem. Eng. Sci. 66, 2554-2567.

Sen, D., Roy, W., Das, L., Sadhu, S., Bhattacharjee, C., 2010. Ultrafiltration of macromolecules using rotating disc membrane module (RDMM) equipped with vanes: Effects of turbulence promoter. J. Membr. Sci. 360, 40-47.

Serra, C.A., Wiesner, M.R., 2000 A comparison of rotating and stationary membrane disk filters using computational fluid dynamics. J. Membr. Sci. 165, 19-29.

Shan, W., 2010. The environmental applications and implications of nanotechnology in membrane-based separations for water treatment, Civil and Environmental Engineering. Michigan State University, East Lansing.

Shan, W., Gaustad, M., Rieck, R., Bénard, A., Petty, C.A., Tarabara, V.V., 2010. Crossflow filtration hydrocyclone for produced water treatment, International Water Association Regional Conference on Membrane Technology and Water Reuse, Istanbul, Turkey, p. 532.

Sherwood, J.D., 1988. The force on a sphere pulled away from a permeable half-space. Physsicochem. Hydrodyn. 10, 3-12.

Sherwood, T.K., Brian, P.L.T., Fisher, R.E., 1967. Desalination by reverse osmosis. I \& EC Fundamentals February 1, 2-12.

Svarovsky, L., Thew, M.T., 1992. Hydrocyclones: Analysis and Applications, Fluid Mechanics and Its Applications. Springer.

Tarabara, V.V., Andre Benard, A., Bruening, M.L., Hogg, S., Ji, P., Motin, A., Shan, W., Tummons, E.N., 2013. Affinity-based hydrocyclone filter for oil-water separation and oil spill cleanup, Gulf of Mexico Oil Spill and Ecosystem Science Conference, New Orleans, LA.

TORR Canada's presentation, $14^{\text {th }}$ International Petroleum Environmental Conference, Houston Texas, 2007.

Tu, Z., Ding, L., 2010. Microfiltration of mineral suspensions using a MSD module with rotating ceramic and polymeric membranes. Separ. Purif. Technol. 73, 363-370. 
van der Sman, R.G.M., Vollebregt, H.M., Mepschen, A., Noordman, T.R., 2012. Review of hypotheses for fouling during beer clarification using membranes. J. Membr. Sci. 396, 22-31.

Vasseur, P., Cox, R.G., 1976. The lateral migration of spherical particle in twodimensional shear flows. 78 .

Viadero Jr, R.C., Masciola, D.A., Reed, B.E., Vaughan Jr., R.L., 2000. Two-phase limiting flux in high-shear rotary ultrafiltration of oil-in-water emulsions. J. Membr. Sci. $175,85-96$.

Vigo, F., Uliana, C., Lupino, P., 1985. The performance of a rotating module in oily emulsions ultrafiltration. Separ. Sci. Technol. 20, 213-230.

Vyas, H.K., Bennett, R.J., Marshall, A.D., 2001. Cake resistance and force balance mechanism in the crossflow microfiltration of lactalbumin particles. J. Membr. Sci. 192, 165-176. 

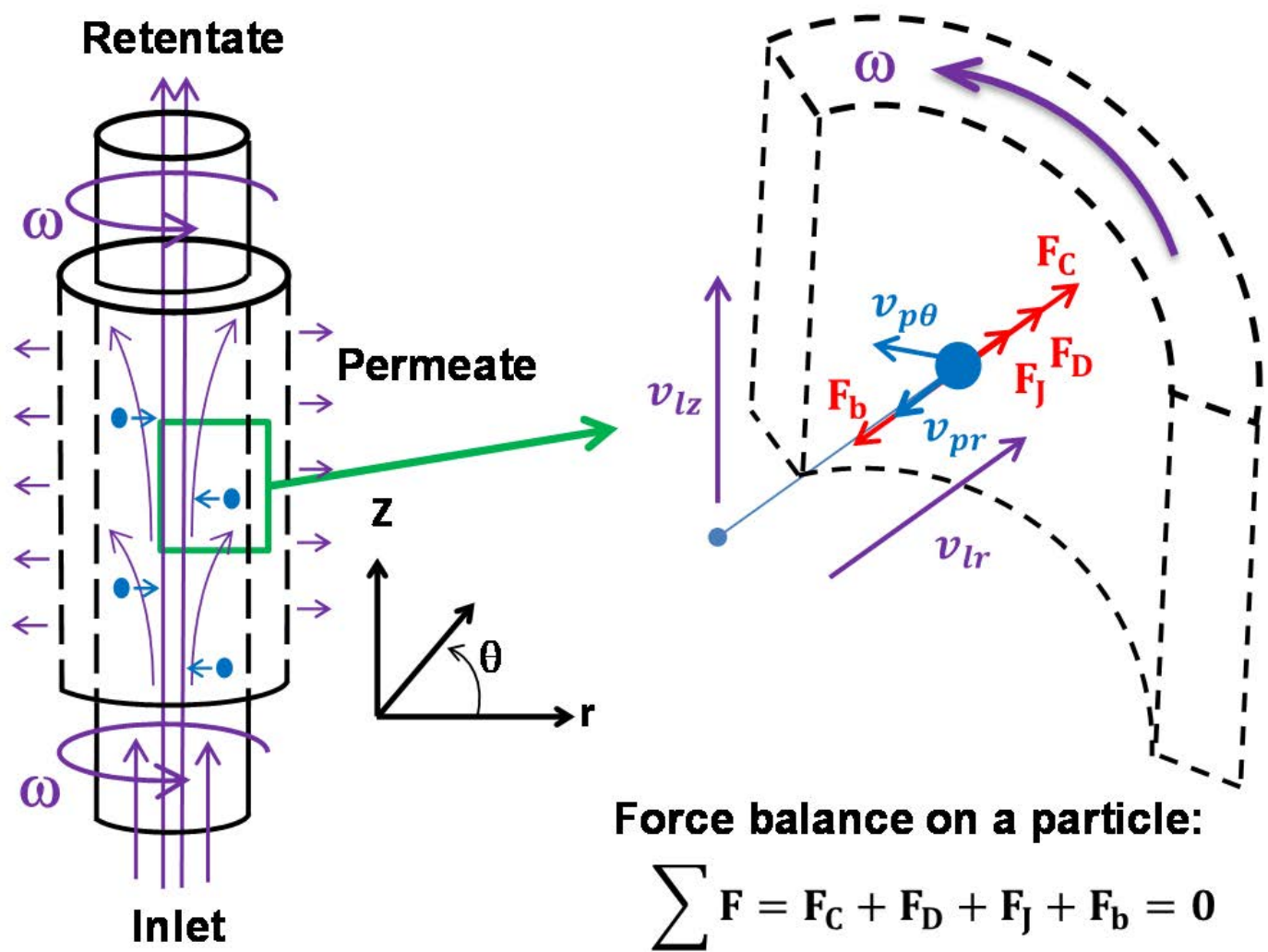

Force balance on a particle:

$$
\sum \mathbf{F}=\mathbf{F}_{\mathbf{C}}+\mathbf{F}_{\mathrm{D}}+\mathbf{F}_{\mathrm{J}}+\mathbf{F}_{\mathbf{b}}=\mathbf{0}
$$

